\title{
Analytical sensitivity analysis using the extended finite element method in shape optimization of bimaterial structures
}

\author{
Lise Noël, Laurent Van Miegroet, Pierre Duysinx* \\ University of Liège - Departement of Aerospace and Mechanical Engineering, Allée de la Découverte 13A, B52, 4000 \\ Liège, Belgium
}

\begin{abstract}
SUMMARY
The present work investigates the shape optimization of bimaterial structures. The problem is formulated using a level set description of the geometry and the extended finite element method (XFEM) to enable an easy treatment of complex geometries. A key issue comes from the sensitivity analysis of the structural responses with respect to the design parameters ruling the boundaries. Even if the approach does not imply any mesh modification, the study shows that shape modifications lead to difficulties when the perturbation of the level sets modifies the set of extended finite elements. To circumvent the problem, an analytical sensitivity analysis of the structural system is developed. Differences between the sensitivity analysis using FEM or XFEM are put in evidence. To conduct the sensitivity analysis, an efficient approach to evaluate the so-called velocity field is developed within the XFEM domain. The proposed approach determines a continuous velocity field in a boundary layer around the zero level set using a local finite element approximation. The analytical sensitivity analysis is validated against the finite differences and a semianalytical approach. Finally our shape optimization tool for bimaterial structures is illustrated by revisiting the classical problem of the shape of soft and stiff inclusions in plates. Copyright (c) 2010 John Wiley \& Sons, Ltd.
\end{abstract}

Received ...

KEY WORDS: shape optimization, analytical sensitivity analysis, XFEM, level set, bimaterial

\section{INTRODUCTION}

There is a general interest in developing optimization tools dealing efficiently with multiphase materials. Advanced shape and topology optimization capabilities are necessary to investigate adequately challenging problems such as designing multimaterial high performance structural components or designing novel multiphase materials with prescribed or extreme properties.

There is little evidence in the literature of successful application of classical shape optimization to design multiphase structures and microstructures. Shape optimization methods were mainly developed in the nineties, but the technology still faces difficulties to address industrial problems. In fact, state-of-the-art shape optimization approach is based on parametric computer aided design (CAD) models and takes advantages of modern automatic meshing tools. However, the approach still suffers from a lack of robustness mostly related to the large modifications of the boundaries leading to major modifications of the mesh, to the degeneracy of the CAD model definition and to possible severe finite element errors after several redesign steps. Therefore, shape optimization requires frequent remeshing operations intended to maintain the conformity of the mesh through

\footnotetext{
${ }^{*}$ Correspondence to: Lise Noël,

University of Liege - Departement of Aerospace and Mechanical Engineering,

Allée de la Découverte 13A, B52, 4000 Liège, Belgium

Lise.Noel@ulg.ac.be
}

Copyright (C) 2010 John Wiley \& Sons, Ltd.

Prepared using nmeauth.cls [Version: 2010/05/13 v3.00] 
the simulation. These operations are extremely costly and time consuming. Furthermore, shape sensitivity analysis requires the resolution of the so-called velocity field problem describing the perturbation of the nodal positions with respect to modifications of the boundaries.

With the seminal work by Bendsøe and Kikuchi [7], topology optimization experienced an enormous expansion taking benefit of a huge research effort to extend its scope to various application fields while simultaneously experiencing a growing impact in industrial applications. Even if voidsolid problems have received most of the attention, the literature review reports several applications of topology optimization to design structures made of several materials. Among others, one can point out the design of microstructures by Sigmund and Torquato [26], the reinforcement of structures or compliant mechanisms [37, 35, 21], or the selection of composite materials from a discrete catalog [23].

In 1988, Osher and Sethian [20] developed the level set method, offering a flexible formalism to deal with moving boundaries. The latters are described implicitly and the method allows an easy handling of shape modifications. Very early, the method was exploited in optimization by Allaire et al. [2] and by Wang et al. [34] and provided an alternative to the widely used Simply Isotropic Material with Penalization (SIMP) description [8]. The level set method offers the opportunity to represent complex geometries with few design parameters, to handle easily large shape modifications and to allow merging and disappearance of certain geometrical entities. Moreover the implicit description of the geometry overcomes some restrictions encountered when implementing the SIMP approach or the explicit description used in CAD. Therefore, the level set method was widely exploited to perform shape and topology optimization in many different ways as referred in the review by Van Dijk et al. [27]. Among other applications, shape and topology optimization using the level set method was successfully applied to design structures involving multiple materials. For instance, Wang and Wang [33] considered the optimal distribution of several material phases in a reference volume using colored level sets. Guo et al. [15] extends the work to the design of multiphase structures subject to local stress constraints while, in Chen et al. [11], the level set method is used to design multiphase energy harvesting devices. More recently Vermaak et al. [31] also investigated the impact of non-monotonous effective properties in multiphase interfaces over the optimal topology of multimaterial structures.

In 1999, Moës et al. [19] proposed a finite element method for crack propagation without remeshing, known as the extended finite element method (XFEM). The method allows taking into account particular behaviors and discontinuities within the elements using an appropriate enrichment of the approximation fields. This approach greatly reduces the difficulty of considering moving boundaries and complex geometries since fixed meshes are used. Taking advantage of the capabilities of the method, difficulties related to remeshing operations, inherent to classical shape optimization, can be mitigated. In addition, the XFEM can be advantageously combined with the level set representation to offer a flexible description of complex geometries as shown in Sukumar et al. [24], Belytschko et al. [6] or Moës et al. [18]. Van Miegroet and Duysinx [30] proposed a generalized shape optimization tool based on a parametric level set description and XFEM. The approach proved to be flexible and efficient in solving 2D and 3D optimization problems [29]. Soon, this opportunity was exploited to perform structural topology optimization as in Li et al. [16] or Wei et al. [36]. Some enhanced XFEM scheme were developed to deal with small feature size by Makhija and Maute [17].

The present research is continuing along and extending the works initiated by Van Miegroet and Duysinx using both XFEM and a level set description to tackle the shape optimization of structures exhibiting interfaces between two or several materials. In fact, there is a great interest in using a flexible geometric description combined with a non-conforming analysis in shape optimization as it enables to consider complex design problems involving local stress and manufacturing constrains. As suggested in works by Van Migroet [29] or by Rotthaus and Barthold [22], carrying out an efficient and rigorous sensitivity analysis is not trivial as using finite perturbation of the level set can lead to modifications of the enrichment field. To circumvent these difficulties in the most general way, they suggested to use analytical derivatives. Some approaches to perform analytical derivatives with XFEM were proposed. Barthold and Materna [3] used a modified XFEM scheme to evaluate 
the design sensitivity analysis. In the fracture mechanics field, Waisman [32] also developed an analytical derivative of the stiffness to extract crack tip strain energy release rates.

Evaluating the derivatives analytically, the key issue of the sensitivity analysis is the determination of a velocity field describing the material flow around the boundaries. The velocity field computation is a classical problem in shape optimization that was investigated within many research works. Besides finite difference methods relying on meshing tools, several approaches to evaluate the velocity field are reported in the literature and summarized in Choi and Chang [12] and Choi and Kim [13]. Among those, one can mention approaches in which the velocity field is naturally determined since it is directly related to the curvilinear coordinates of Bezier or B-Spline curves used to represent the moving boundaries as in Braibant and Fleury [10], [9]. Velocity fields can also be determined by solving an auxiliary problem with prescribed forces as in the fictitious load method by Belegundu and Rajan [5] or with prescribed displacements as in the boundary displacement method by Beckers [4].

The idea of this paper is to tackle the optimization of bimaterial structures and microstructures within an XFEM-level set framework. Dealing with material interfaces, the enrichment functions used to create the extended approximation field are no longer the same as the ones used for voidmaterial interfaces. Therefore, at first the paper shows that the sensitivity analysis has to be adapted and then, focuses on the theoretical development and the implementation of the sensitivity analysis using an analytical approach. In particular, the derivative of the stiffness matrix of the structures is a key central issue. Starting from its discretized expression, the derivative of the stiffness matrix is obtained by deriving with respect to the design parameters. Once this analytical expression of the stiffness matrix derivative is available, the sensitivity of various objective and constraint functions, such as the compliance, the displacements or the local stresses, can be easily evaluated.

The paper proceeds as follow. The XFEM-level set framework exploited in this paper is recalled in section 2 . Then, different methods are proposed to perform the sensitivity analysis in section 3 . The section focuses on the problems encountered using classical methods, such as the finite differences, in the bimaterial XFEM-level set framework. Then, the analytical method is introduced. In section 4 , the analytical sensitivity analysis is developed and explained in details. The computation of the derivatives requires the evaluation of a velocity field. Section 5 focuses on the original approach developed to evaluate the velocity field exploiting the level set description of the geometry. In section 6 , the analytical sensitivity analysis is tested and validated against classical methods on academic examples. Finally, the sensitivity analysis is illustrated by solving a classical shape optimization benchmark, revisiting the optimal shape of an elliptical stiff or soft inclusion in a plate.

\section{OPTIMIZATION FRAMEWORK}

\subsection{Problem formulation}

This work focuses on the evaluation of the sensitivity analysis necessary to solve optimization problems such as finding the optimal geometry of elastic structures made of two materials. Two different materials are distributed within a given design domain to minimize some objective while satisfying some prescribed design constraints. The generic statement of the optimization problem to solve is given as follow:

$$
\begin{array}{ll}
\min _{\mathbf{s}} & g_{0}(\mathbf{x}, \mathbf{s}) \\
\text { s.t. } & g_{j} \leq 0, \quad j=1, \ldots, n_{j} \\
& \underline{s}_{i} \leq s_{i} \leq \bar{s}_{i}, \quad i=1, \ldots, n_{i}
\end{array}
$$

where $g_{0}$ is some objective function, $g_{j}$ some given constraint, $\mathbf{x}$ the space variables and $\mathbf{s}$ the set of design parameters.

To achieve this goal, the XFEM is combined with a level set description of the geometry. The basic principles of these methods, necessary for further developments in this paper, are recalled in the following sections. 


\subsection{Level set description of geometry}

A level set description is used to represent the geometry of the boundaries within the design domain. A level set function $\phi$ describes the repartition of the materials over the design domain. The level set function is dependent of the design parameters s:

$$
\phi=\phi(\mathbf{x}, \mathbf{s}) .
$$

The most common level set function is the signed distance function. The latter is used through this work. In general, the material A domain is identified by positive values of the level set function $\phi$ and the material B domain lies where $\phi$ is negative:

$$
\begin{cases}\phi(\mathbf{x}, \mathbf{s})>0, & \forall \mathbf{x} \in \Omega_{A} \\ \phi(\mathbf{x}, \mathbf{s})=0, & \forall \mathbf{x} \in \Gamma_{A B} \\ \phi(\mathbf{x}, \mathbf{s})<0, & \forall \mathbf{x} \in \Omega_{B}\end{cases}
$$

where $\Omega_{A}$ is the domain filled with material A, $\Omega_{B}$ the domain filled with material B and $\Gamma_{A B}$ the interface between the two materials A and B, as depicted in Figure 1.

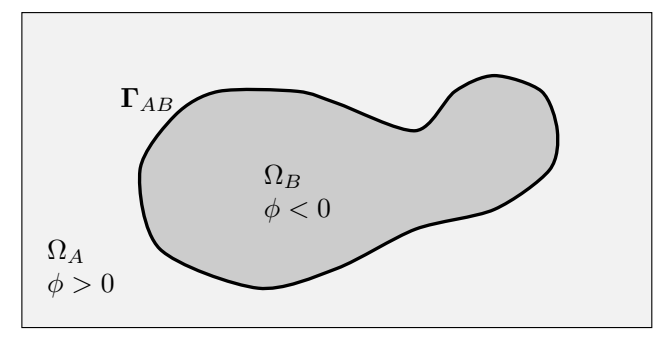

Figure 1. Repartition of materials on the domain $\Omega$ using a level set function.

Conversely to the works by Allaire et al. [2] and Wang et al. [34], where the level set function is updated by solving the Hamilton-Jacobi equation, here the level set function is updated through the evolution of the design parameters s using mathematical programming tools.

\subsection{The extended finite element method}

The response of the elastic structures is obtained using the XFEM. This method allows taking into account discontinuous behaviors within the elements by adding specific shape functions to the classical finite element approximation field:

$$
u^{h}(\mathbf{x})=\underbrace{\sum_{i \in I} N_{i}(\mathbf{x}) u_{i}}_{\text {FEM }}+\underbrace{\sum_{i \in I^{\star}} N_{i}^{\star}(\mathbf{x}) a_{i}}_{\text {Enrichment }},
$$

where $I$ is the set of all the mesh nodes, $N_{i}(\mathbf{x})$ are the standard finite element shape functions, $u_{i}$ are the degrees of freedom associated to the standard shape functions $N_{i}(\mathbf{x}), I^{\star}$ is the set of enriched nodes, $N_{i}^{\star}(\mathbf{x})$ are the enriched shape functions and $a_{i}$ are the additional unknowns related to the enrichment. The enrichment is local and is only used for the elements that have to represent the discontinuities, i.e. the elements crossed by the interface as shown in Figure 2.

Combining the XFEM and the level set description of geometry, the material interface is directly represented by the iso-zero level set and there is no need to introduce any additional field of density to describe the interface, as in the SIMP approach. Working on a discretized mesh, the level set function is conveniently represented using its nodal values and interpolated using classical finite element shape functions:

$$
\phi^{h}(\mathbf{x})=\sum_{i} N_{i}(\mathbf{x}) \phi_{i}
$$




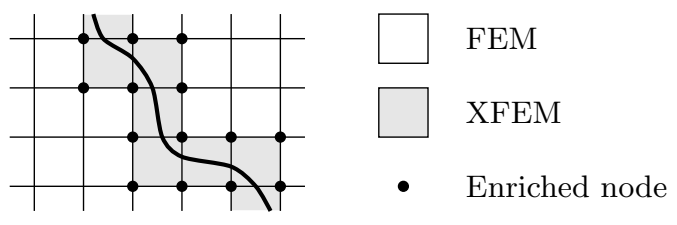

Figure 2. Selection of the enriched nodes and elements on a mesh.

\subsection{XFEM for material interfaces}

This work focuses on discontinuities related to interfaces between two materials exhibiting different properties. Interfaces between materials are characterized by a continuous displacement field, but they introduce a discontinuity in the strain field. These kinds of discontinuity are commonly treated using the ridge function introduced by Moës [18] as enrichment function. This particular enrichment function $\psi$ is used in this work and is given as:

$$
\psi(\mathbf{x})=\sum_{i} N_{i}(\mathbf{x})\left|\phi_{i}\right|-\left|\sum_{i} N_{i}(\mathbf{x}) \phi_{i}\right| .
$$

The enriched shape function $N_{i}(\mathbf{x})^{\star}$ are built by multiplying the classical finite element shape functions $N_{i}(\mathbf{x})$ by the enrichment function $\psi(\mathbf{x})$ :

$$
N_{i}(\mathbf{x})^{\star}=\psi(\mathbf{x}) N_{i}(\mathbf{x}) .
$$

Proceeding this way, the obtained enriched function $N_{i}^{\star}(\mathbf{x})$ presents terms up to the power two for bars or linear T3 triangles and up to the power four for bilinear Q4 quadrangles. Therefore, to capture precisely the discontinuity behavior, a suitable number of Gauss points is necessary to perform the integration over the elements. An investigation of the influence of the number of Gauss points used is performed later in Section 6.

\subsection{XFEM implementation}

This section is devoted to the exploitation of the XFEM to tackle the problem of material interfaces. Some implementation details are reminded and will be used in the coming developments.

2.5.1. Element subdivision The location of the interface is given by the iso-zero level set and the level set function is used to perform the subdivision of the element into a subdomain filled with material A and a subdomain filled with material B.

For the sake of simplicity, the level set function is approximated by a straight line when searching for the intersections between the material interface and the elements edges as shown in Figure 3. In 2D, the coordinates of the intersection $\left(\xi^{\star}, \eta^{\star}\right)$ are easily determined using the level set nodal values $\phi_{1}, \phi_{2}$ and the nodal coordinates $\left(\xi_{1}, \eta_{1}\right),\left(\xi_{2}, \eta_{2}\right)$ :

$$
\left\{\begin{array}{l}
\xi^{\star}=t \xi_{2}+(1-t) \xi_{1} \\
\eta^{\star}=t \eta_{2}+(1-t) \eta_{1}
\end{array} \quad \text { with } \quad t=\frac{\left|\phi_{1}\right|}{\left|\phi_{1}\right|+\left|\phi_{2}\right|} .\right.
$$

2.5.2. Integration Working with XFEM, some elements are filled with two different materials. For those elements, integration requires some particular care because of the strain field discontinuity that exists at the interface. Integration can no longer be performed using a classical Gauss quadrature. In fact, the obtained results would not be satisfactory even if resorting to a tremendous amount of Gauss points. To perform an accurate integration, each part of an element across the material interface is treated independently by introducing an integration mesh on XFEM elements. Dividing the parent 


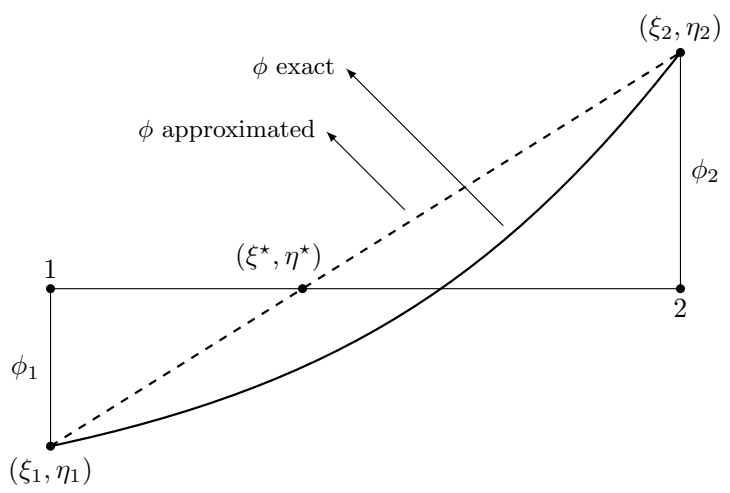

Figure 3. Computation of the intersection between the level set function and the element edges in 2D.

element into subdomains in a first parametric space $(\xi, \eta)$ and mapping these subdomains to a second parametric space $(l, m)$, a sufficient number of suitably located Gauss points is obtained. The classical Gauss quadrature procedure can anew be applied. This integration procedure is illustrated in Figure 4.
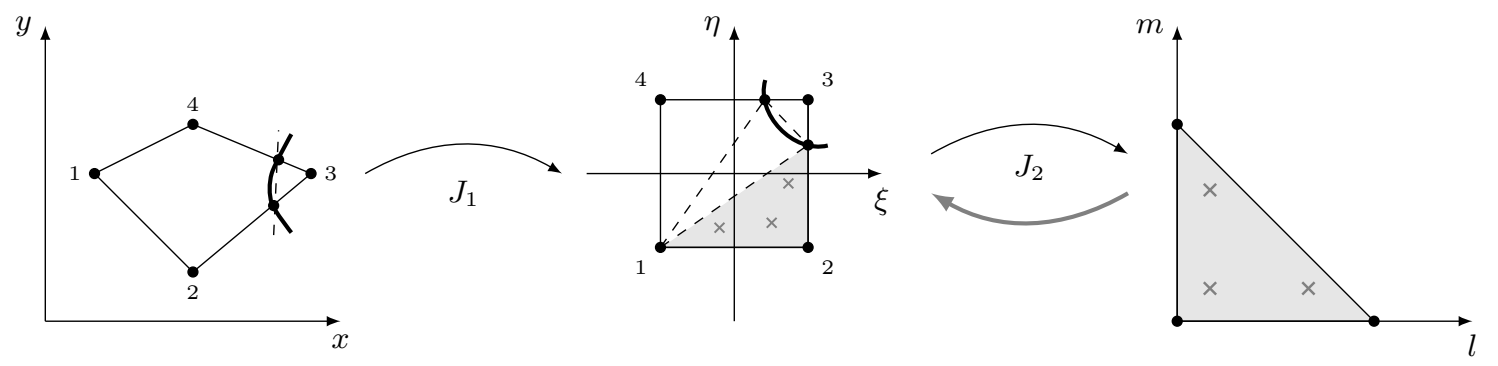

Figure 4. Integration procedure for extended finite elements illustrated on a quadrangle. The quadrangular element is mapped to a parent reference element using a first mapping $J_{1}$. The interface is approximated by a straight line to carry out the subdivision of the parent element. The subdomains of this element are mapped to a second reference space based on a set of mappings $J_{2}$. Gauss points are then brought back from the second reference space to the first one, where classical Gauss quadrature can be performed.

In this work, the integration procedure detailed before is mainly exploited for the evaluation of the stiffness matrix $\mathbf{K}$. The elementary stiffness matrix is computed for each element by adding the contribution of all the Gauss points:

$$
\begin{aligned}
\mathbf{K}_{e} & =\int_{\Omega_{e}}\left[\begin{array}{l}
\mathbf{B}_{u}^{T} \\
\mathbf{B}_{a}^{T}
\end{array}\right] \mathbf{H}\left[\begin{array}{ll}
\mathbf{B}_{u} & \mathbf{B}_{a}
\end{array}\right] d \Omega_{e} \\
& =\int_{\Omega_{e}}\left[\begin{array}{c}
\mathbf{B}_{u}^{T} \\
\mathbf{B}_{a}^{T}
\end{array}\right] \mathbf{H}\left[\begin{array}{cc}
\mathbf{B}_{u} & \mathbf{B}_{a}
\end{array}\right]\left|\mathbf{J}_{1}\right|\left|\mathbf{J}_{2}\right| d \xi d \eta \\
& \simeq \sum_{g p} w_{g p}\left[\begin{array}{l}
\mathbf{B}_{u}^{T} \\
\mathbf{B}_{a}^{T}
\end{array}\right]_{g p} \mathbf{H}_{g p}\left[\begin{array}{ll}
\mathbf{B}_{u} & \mathbf{B}_{a}
\end{array}\right]_{g p}\left|\mathbf{J}_{1}\right|_{g p}\left|\mathbf{J}_{2}\right|_{g p},
\end{aligned}
$$

where $\Omega_{e}$ is the element domain, $\sum_{g p}$ is the sum over all the element Gauss points, $\mathbf{B}_{u}$ is the part of the $\mathbf{B}$ matrix of the derived shape functions related to the classical degrees of freedom $u_{i}, \mathbf{B}_{a}$ is the 
part of the $\mathbf{B}$ matrix of the derived shape functions related to the additional degrees of freedom $a_{i}, \mathbf{H}$ is the Hooke's matrix, $\left|\mathbf{J}_{1}\right|$ is the determinant of the first Jacobian mapping, $\left|\mathbf{J}_{2}\right|$ is the determinant of the second Jacobian mapping, $w_{g p}$ are the weights associated to Gauss points.

\section{DESIGN SENSITIVITY ANALYSIS}

Several approaches can be exploited to carry out the sensitivity analysis as reviewed in Van Keulen et al. [28] or Adelman and Haftka [1]. The paper aims at comparing these approaches when applied to an XFEM-level set framework with material interfaces. In particular, an analytical approach is developed. This analytical approach, although more complex, is highly appropriate and efficient in the specific framework that is considered. It will be showed that it offers the most general and robust approach in practice.

\subsection{Global finite difference}

A first approach, that is the easiest and the most straightforward to implement, is the global finite difference over the objective $g_{0}$ and constraint functions $g_{j}$. This approach can be widely used to solve any optimization problems:

$$
\begin{aligned}
\frac{d g_{0}}{d s} \simeq \frac{g_{0}(s+\delta s)-g_{0}(s)}{\delta s} \\
\frac{d g_{j}}{d s} \simeq \frac{g_{j}(s+\delta s)-g_{j}(s)}{\delta s} .
\end{aligned}
$$

However, with an XFEM-level set framework, some problems can occur when the interface is perturbed. As the geometry is slightly modified, some elements can undergo status changes, i.e. they can change their status from cut (resp. uncut) by the interface to uncut (resp. cut), and so change their nature from XFEM (resp. FEM) to FEM (resp. XFEM). At the occurrence of changes of element status, the number of degrees of freedom associated to the structures as well as the approximation fields used are modified. These modifications lead to the introduction of errors in the finite difference computation of the derivatives.

\subsection{Discrete semi-analytical and analytical sensitivity}

The sensitivity analysis can be performed on the discretized system $\mathbf{K u}=\mathbf{f}$ where $\mathbf{K}$ is the global stiffness matrix, $\mathbf{u}$ the vector of the generalized displacements and $\mathbf{f}$ the vector of the external forces. After discretizing the governing continuum equations, one derives these with respect to the design parameters s. In the particular case of linear elastic problems and deriving with respect to a particular design parameter $s$, the procedure leads to the following expression:

$$
\frac{d \mathbf{u}}{d s}=\mathbf{K}^{-1}\left(\frac{\partial \mathbf{f}}{\partial s}-\frac{\partial \mathbf{K}}{\partial s} \mathbf{u}\right),
$$

where $\frac{d}{d s}$ and $\frac{\partial}{\partial s}$ are respectively the total and the partial derivatives with respect to $s$. This equation allows evaluating the derivative of the vector of the generalized displacements $\mathbf{u}$ with respect to the considered design parameter $s$, provided that the derivatives of the stiffness matrix $\mathbf{K}$ and of the vector of external forces $\mathbf{f}$ can be computed.

In this work, the external forces are considered independent of the design parameters $\mathbf{s}$, so its derivative is equal to zero. The following sections focus on tailoring a convenient and efficient approach to evaluate the stiffness matrix derivative.

3.2.1. Semi-analytical approach A first approach is obtained combining Eq.(11) and a finite difference computation of the derivative of the elementary stiffness matrices, as given in Eq.(12). This approach is known as the semi-analytical method. The latter was successfully implemented 
to solve shape optimization problems within an XFEM-level set framework for void-material interfaces by Van Miegroet et al. [29]. This method can be extended to the case of materials interfaces.

$$
\frac{\partial \mathbf{K}_{e}}{\partial s} \simeq \frac{\mathbf{K}_{e}(s+\delta s)-\mathbf{K}_{e}(s)}{\delta s}
$$

where $\mathbf{K}_{e}$ is the stiffness matrix of element e.

As previously mentioned for the global finite difference method, some problems arise as element status changes occur during finite perturbation of the boundary. In this case, as the number of degrees of freedom is modified, a finite difference on the stiffness matrices can no longer be carried out since the dimensions, before and after interface perturbation, mismatch.

In the case of void-material interfaces, Van Miegroet and Dusyinx [30] apply a strategy called the ignore strategy. The latter consists in ignoring the contribution of elements undergoing a status change, introducing an error in the computation of the derivative.

Several strategies were developed to prevent the changes of the elements status. It mainly consists in some preprocessing operations aiming at applying a convenient interface perturbation. Three of these methods are explained below and illustrated in Figure 5.

A first strategy consists in perturbing the interface forward and backward to check whether a change of element status occurs or not. If not, a central finite difference can be applied to evaluate the derivatives. If there is a change of element status while moving the interface forward (resp. backward), a backward (resp. forward) finite difference is used. This strategy requires extra computation to determine which kind of finite differencing scheme should be performed.

A second strategy consists in perturbing the interface forward or backward to insure that no element initially cut by the interface becomes uncut or vice versa. If some elements undergo a status change, the mesh nodes are relocated so that the interface perturbation does not lead to any status change anymore. However, relocating the mesh nodes weakens the advantages of using XFEM, especially implemented to work on fixed meshes and to avoid remeshing operations.

A third strategy consists in perturbing the interface forward and backward to know if the interface is going out of the elements. If so, a maximum perturbation size is determined. This perturbation size is adapted to be sufficiently small so that no change of element status occurs. This strategy exhibits an inherent drawback as the perturbation size can become so small that it could introduce round-off errors and lead to errors in the derivative evaluation.

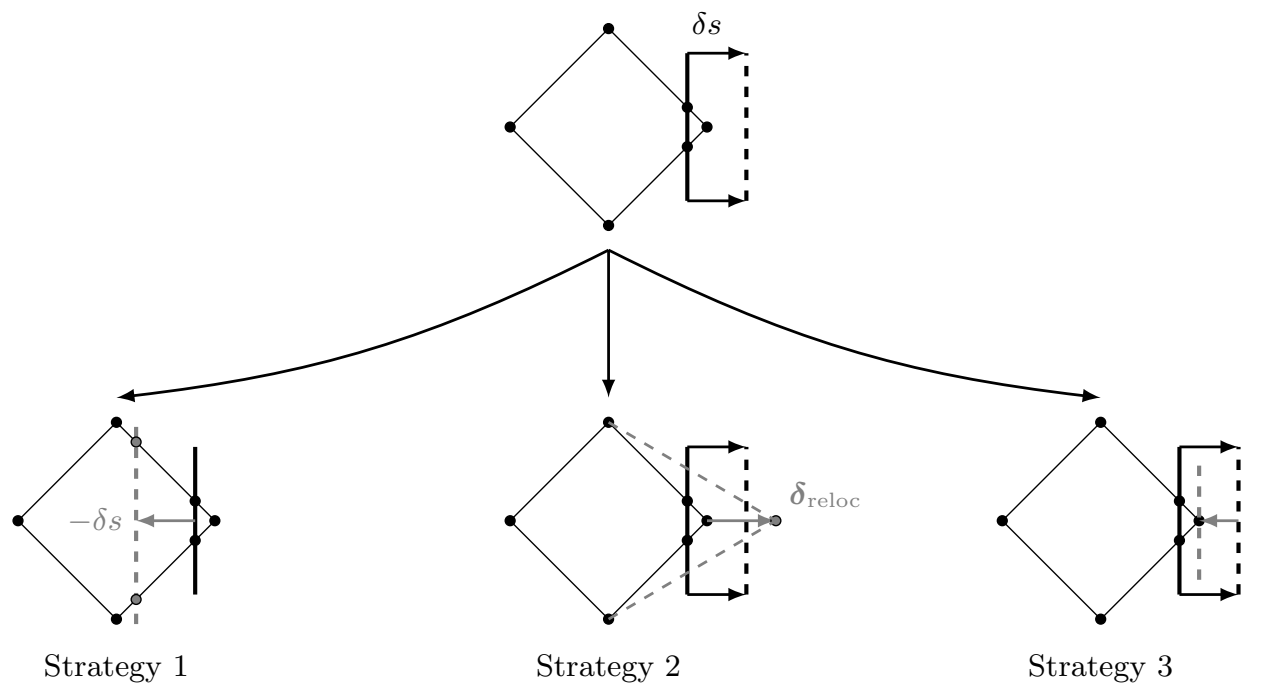

Figure 5. Preprocessing strategies to avoid element status change. 
Preprocessing operations imply additional computational time and so a potential CPU time penalty. To avoid preprocessing operations, an analytical approach is developed to perform the sensitivity analysis and is considered in the following sections.

3.2.2. Analytical approach To circumvent the problems related to the change of element status and to avoid any preprocessing operations or remeshing operations, the stiffness matrix derivative can be evaluated analytically. Although this method is more complex and difficult to implement, it exhibits several advantages over the previous approaches since no interface perturbation is required.

The analytical derivative expression of the stiffness matrix can be obtained starting from its discretized expression, as written in Eq.(13). The Hooke's matrix $\mathbf{H}$ is assumed independent of the design parameters s. For the sake of simplicity, the subscripts and the dependence on the design parameters are dropped in coming developments.

$$
\mathbf{K}=\sum_{g p} w_{g p}\left[\begin{array}{l}
\mathbf{B}_{u}(\mathbf{s})^{T} \\
\mathbf{B}_{a}(\mathbf{s})^{T}
\end{array}\right]_{g p} \mathbf{H}_{g p}\left[\begin{array}{ll}
\mathbf{B}_{u}(\mathbf{s}) & \mathbf{B}_{a}(\mathbf{s})
\end{array}\right]_{g p}\left|\mathbf{J}_{1}(\mathbf{s})\right|_{g p}\left|\mathbf{J}_{2}(\mathbf{s})\right|_{g p} .
$$

Deriving Eq.(13) with respect to a design parameter $s$, the following expression is found:

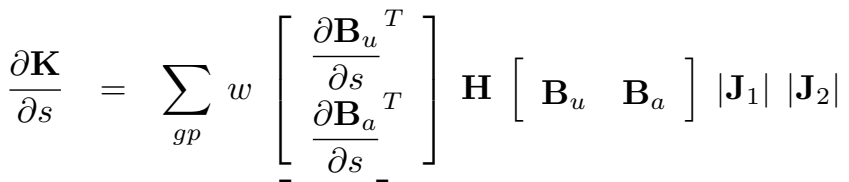

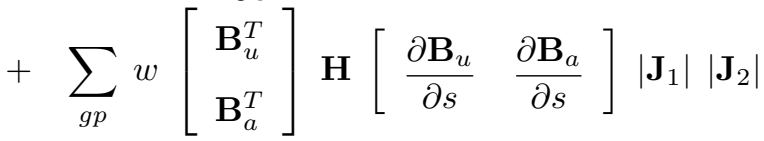

$$
\begin{aligned}
& +\sum_{g p} w\left[\begin{array}{c}
\mathbf{B}_{u}^{T} \\
\mathbf{B}_{a}^{T}
\end{array}\right] \mathbf{H}\left[\begin{array}{ll}
\mathbf{B}_{u} & \mathbf{B}_{a}
\end{array}\right] \frac{\partial\left|\mathbf{J}_{1}\right|}{\partial s}\left|\mathbf{J}_{2}\right| \\
& +\sum_{g p} w\left[\begin{array}{c}
\mathbf{B}_{u}^{T} \\
\mathbf{B}_{a}^{T}
\end{array}\right] \mathbf{H}\left[\begin{array}{ll}
\mathbf{B}_{u} & \mathbf{B}_{a}
\end{array}\right]\left|\mathbf{J}_{1}\right| \frac{\partial\left|\mathbf{J}_{2}\right|}{\partial s} .
\end{aligned}
$$

The main issue is now to determine the derivatives of the $\mathbf{B}$ matrices $\frac{\partial \mathbf{B}_{u}}{\partial s}, \frac{\partial \mathbf{B}_{a}}{\partial s}$ and of the determinants of the Jacobian mappings $\frac{\partial\left|\mathbf{J}_{1}\right|}{\partial s}, \frac{\partial\left|\mathbf{J}_{2}\right|}{\partial s}$.

\section{ANALYTIC SENSITIVITY ANALYSIS}

This section details the expressions needed to perform the analytical sensitivity analysis taking into account the particularities of working with XFEM.

\subsection{Derivatives with XFEM}

The perturbation of the interface, i.e. the perturbation of a design parameter $s$, does not lead to the same situation while working with XFEM or FEM. With XFEM, since the mesh is fixed, parameter perturbations do not modify the geometry of the mesh in the physical space. However, it modifies the respective volumes allocated to each material since it changes the integration mesh used on XFEM elements. The derivative expression of the stiffness matrix is thus different if working with FEM or XFEM. The particularities related to the XFEM are detailed in the schematic representation sketched in Figure 6.

In the physical space $(x, y)$, the perturbation of a design parameter $s$ does not lead to any mesh modifications. Thereby, the nodal coordinates $(x, y)$ in the physical space remain independent of the design parameter $s$. 
As an element in the physical space $(x, y)$ remains unchanged, it is mapped to the same reference element in the first parametric space $(\xi, \eta)$ regardless of the interface perturbation. Therefore, the first Jacobian mapping $\mathbf{J}_{1}$ is independent of the design parameters and the shape functions $N_{i}(\xi, \eta)$ remain unchanged. However, the subdivision of the reference element into subdomains is modified and the coordinates $(\xi, \eta)$ of a point interior to the parent element in the first parametric space are dependent of the design parameter.

Subdomains from the initial and perturbed configurations are mapped to the same reference element in the second parametric space $(l, m)$, even though they are different. Thus, the second Jacobian mapping $\mathbf{J}_{2}$ is different and specific for each subdomain and is sensitive to the design parameters. Since the reference element remains the same, the shape functions $N_{i}(l, m)$ are not modified. The coordinates $(l, m)$ in the second parametric space are also independent of the design parameters.

Dealing with material interfaces, special enriched shape functions are added to the approximation. These additional shape functions $N_{i}^{\star}=\psi N_{i}$ are dependent of the design parameters and they introduce new terms in the expression of the derivative.

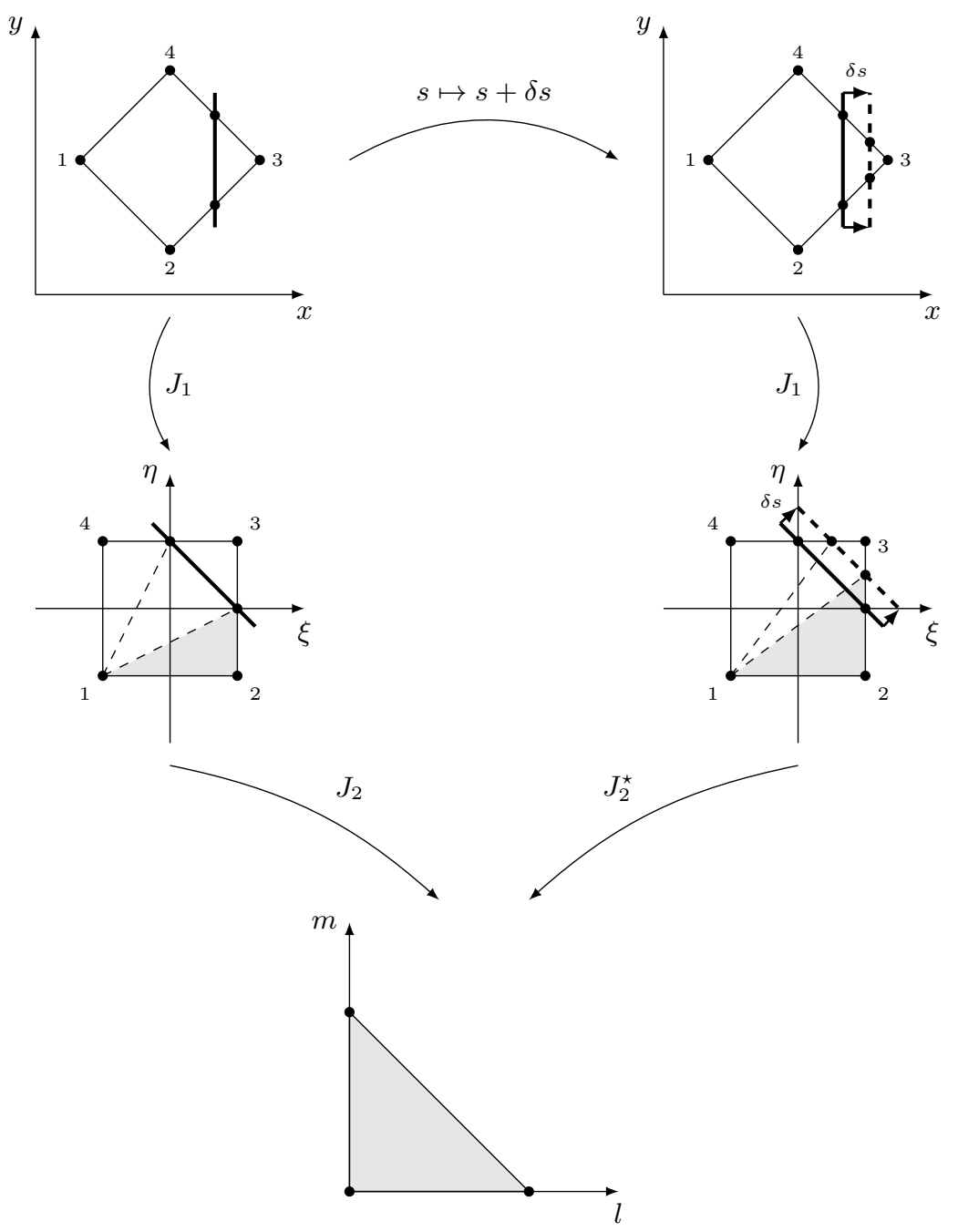

Figure 6. Computation of analytical derivatives within an XFEM framework.

Finally, working with XFEM, the derivative of the stiffness matrix is reduced to the expression given in Eq.(15). The two first terms represent the variations of the derivative of the shape functions 
with the design parameter $s$. The third term represents the sensitivity of the integration domains respectively attributed to each material as the design parameter $s$ is modified.

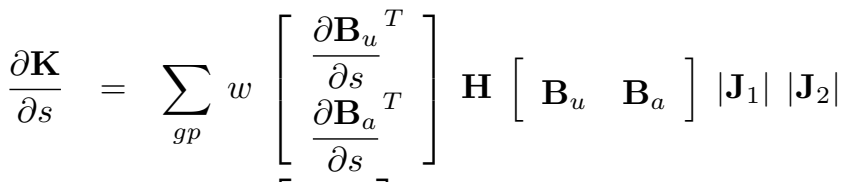

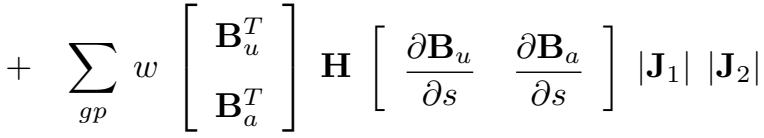

$$
\begin{aligned}
& +\sum_{g p} w\left[\begin{array}{c}
\mathbf{B}_{u}^{T} \\
\mathbf{B}_{a}^{T}
\end{array}\right] \mathbf{H}\left[\begin{array}{ll}
\mathbf{B}_{u} & \mathbf{B}_{a}
\end{array}\right]\left|\mathbf{J}_{1}\right| \frac{\partial\left|\mathbf{J}_{2}\right|}{\partial s} .
\end{aligned}
$$

\subsection{Computational details}

This section of the paper gives all the detailed expressions of the derivatives, focusing on a two dimensional framework for the sake of simplicity.

4.2.1. Derivative of the determinant of the second Jacobian mapping $J_{2}$ The second Jacobian $\mathbf{J}_{2}$ is given by Cook [14]:

$$
\left|\mathbf{J}_{2}\right|=\left(\sum_{i} \frac{\partial N_{i}}{\partial l} \xi_{i}\right)\left(\sum_{i} \frac{\partial N_{i}}{\partial m} \eta_{i}\right)-\left(\sum_{i} \frac{\partial N_{i}}{\partial m} \xi_{i}\right)\left(\sum_{i} \frac{\partial N_{i}}{\partial l} \eta_{i}\right) .
$$

Deriving the equation (16) with respect to the design parameter $s$, one obtains:

$$
\begin{aligned}
\frac{\partial\left|\mathbf{J}_{2}\right|}{\partial s}=\frac{\partial}{\partial s}\left(\sum_{i} \frac{\partial N_{i}}{\partial l} \xi_{i}\right)\left(\sum_{i} \frac{\partial N_{i}}{\partial m} \eta_{i}\right)+\left(\sum_{i} \frac{\partial N_{i}}{\partial l} \xi_{i}\right) \frac{\partial}{\partial s}\left(\sum_{i} \frac{\partial N_{i}}{\partial m} \eta_{i}\right) \\
-\frac{\partial}{\partial s}\left(\sum_{i} \frac{\partial N_{i}}{\partial m} \xi_{i}\right)\left(\sum_{i} \frac{\partial N_{i}}{\partial l} \eta_{i}\right)-\left(\sum_{i} \frac{\partial N_{i}}{\partial m} \xi_{i}\right) \frac{\partial}{\partial s}\left(\sum_{i} \frac{\partial N_{i}}{\partial l} \eta_{i}\right) .
\end{aligned}
$$

Working with XFEM, the derivative with respect to the design parameter $s$ of the first derivatives of the shape functions are equal to zero and the equation is reduced to:

$$
\begin{aligned}
\frac{\partial\left|\mathbf{J}_{2}\right|}{\partial s}= & \left(\sum_{i} \frac{\partial N_{i}}{\partial l} \frac{\partial \xi_{i}}{\partial s}\right)\left(\sum_{i} \frac{\partial N_{i}}{\partial m} \eta_{i}\right)+\left(\sum_{i} \frac{\partial N_{i}}{\partial l} \xi_{i}\right)\left(\sum_{i} \frac{\partial N_{i}}{\partial m} \frac{\partial \eta_{i}}{\partial s}\right) \\
& -\left(\sum_{i} \frac{\partial N_{i}}{\partial m} \frac{\partial \xi_{i}}{\partial s}\right)\left(\sum_{i} \frac{\partial N_{i}}{\partial l} \eta_{i}\right)-\left(\sum_{i} \frac{\partial N_{i}}{\partial m} \xi_{i}\right)\left(\sum_{i} \frac{\partial N_{i}}{\partial l} \frac{\partial \eta_{i}}{\partial s}\right) .
\end{aligned}
$$

The derivative of $\left|\mathbf{J}_{\mathbf{2}}\right|$ can be calculated provided that $\frac{\partial \xi}{\partial s}$ and $\frac{\partial \eta}{\partial s}$ are known. These derivatives describe the sensitivity of nodal positions in the first reference parametric space $(\xi, \eta)$ with respect to the modification of the boundary. Referring to classical literature terminology, $\left(\frac{\partial \xi}{\partial s}, \frac{\partial \eta}{\partial s}\right)$ is the velocity field in the first reference parametric space $(\xi, \eta)$.

Derivative of the FEM part of the B matrix $\mathbf{B}_{u} \quad$ The FEM part of the $\mathbf{B}$ matrix is built using the following terms:

$$
\left[\begin{array}{c}
\frac{\partial N_{i}(\xi, \eta)}{\partial x} \\
\frac{\partial N_{i}(\xi, \eta)}{\partial y}
\end{array}\right]=\mathbf{J}_{1}^{-1}\left[\begin{array}{c}
\frac{\partial N_{i}(\xi, \eta)}{\partial \xi} \\
\frac{\partial N_{i}(\xi, \eta)}{\partial \eta}
\end{array}\right] .
$$


Deriving the equation (19) with respect to the design parameter $s$ and making use of:

$$
d\left(X^{-1}\right)=-X^{-1} d X X^{-1}
$$

one gets:

$$
\frac{\partial}{\partial s}\left[\begin{array}{c}
\frac{\partial N_{i}}{\partial x} \\
\frac{\partial N_{i}}{\partial y}
\end{array}\right]=-\mathbf{J}_{1}^{-1} \frac{\partial \mathbf{J}_{1}}{\partial s} \mathbf{J}_{1}^{-1}\left[\begin{array}{c}
\frac{\partial N_{i}}{\partial \xi} \\
\frac{\partial N_{i}}{\partial \eta}
\end{array}\right]+\mathbf{J}_{1}^{-1}\left[\begin{array}{c}
\frac{\partial^{2} N_{i}}{\partial \xi^{2}} \frac{\partial \xi}{\partial s}+\frac{\partial^{2} N_{i}}{\partial \xi \partial \eta} \frac{\partial \eta}{\partial s} \\
\frac{\partial^{2} N_{i}}{\partial \eta \partial \xi} \frac{\partial \xi}{\partial s}+\frac{\partial^{2} N_{i}}{\partial \eta} \frac{\partial \eta}{\partial s}
\end{array}\right] .
$$

Working with XFEM, the derivative of the first Jacobian $\mathbf{J}_{1}$ is equal to zero and the derivative of the FEM part of the B matrix, as given in Eq.(21), is reduced to the following equation:

$$
\frac{\partial}{\partial s}\left[\begin{array}{c}
\frac{\partial N_{i}}{\partial x} \\
\frac{\partial N_{i}}{\partial y}
\end{array}\right]=\mathbf{J}_{1}^{-1}\left[\begin{array}{c}
\frac{\partial^{2} N_{i}}{\partial \xi^{2}} \frac{\partial \xi}{\partial s}+\frac{\partial^{2} N_{i}}{\partial \xi \partial \eta} \frac{\partial \eta}{\partial s} \\
\frac{\partial^{2} N_{i}}{\partial \eta \partial \xi} \frac{\partial \xi}{\partial s}+\frac{\partial^{2} N_{i}}{\partial \eta^{2}} \frac{\partial \eta}{\partial s}
\end{array}\right]
$$

Similarly to the derivative of $\left|\mathbf{J}_{2}\right|$, the derivative of the FEM part of the $\mathbf{B}$ matrix requires the evaluation of the velocity field $\left(\frac{\partial \xi}{\partial s}, \frac{\partial \eta}{\partial s}\right)$ in the first reference parametric space $(\xi, \eta)$.

4.2.2. Derivative of the enriched part of the $\mathbf{B}$ matrix $\mathbf{B}_{a}$ The XFEM part of the $\mathbf{B}$ matrix is built using the following terms:

$$
\left[\begin{array}{c}
\frac{\partial N_{i}^{\star}(\xi, \eta)}{\partial x} \\
\frac{\partial N_{i}^{\star}(\xi, \eta)}{\partial y}
\end{array}\right]=\mathbf{J}_{1}^{-1}\left(\left[\begin{array}{c}
\frac{\partial N_{i}(\xi, \eta)}{\partial \xi} \\
\frac{\partial N_{i}(\xi, \eta)}{\partial \eta}
\end{array}\right] \psi+\left[\begin{array}{c}
\frac{\partial \psi}{\partial \xi} \\
\frac{\partial \psi}{\partial \eta}
\end{array}\right] N_{i}(\xi, \eta)\right)
$$

Deriving the equation (23) with respect to the design parameter $s$ and given Eq.(20), one gets:

$$
\begin{aligned}
& \frac{\partial}{\partial s}\left[\begin{array}{c}
\frac{\partial N_{i}^{\star}}{\partial x} \\
\frac{\partial N_{i}^{\star}}{\partial y}
\end{array}\right]=-\mathbf{J}_{1}^{-1} \frac{\partial \mathbf{J}_{1}}{\partial s} \mathbf{J}_{1}^{-1}\left(\left[\begin{array}{c}
\frac{\partial N_{i}}{\partial \xi} \\
\frac{\partial N_{i}}{\partial \eta}
\end{array}\right] \psi+\left[\begin{array}{c}
\frac{\partial \psi}{\partial \xi} \\
\frac{\partial \psi}{\partial \eta}
\end{array}\right] N_{i}\right) \\
& +\mathbf{J}_{1}^{-1}\left(\left[\begin{array}{c}
\frac{\partial^{2} N_{i}}{\partial \xi^{2}} \frac{\partial \xi}{\partial s}+\frac{\partial^{2} N_{i}}{\partial \xi \partial \eta} \frac{\partial \eta}{\partial s} \\
\frac{\partial^{2} N_{i}}{\partial \eta^{2}} \frac{\partial \eta}{\partial s}+\frac{\partial^{2} N_{i}}{\partial \eta \partial \xi} \frac{\partial \xi}{\partial s}
\end{array}\right] \psi+\left[\begin{array}{c}
\frac{\partial N_{i}}{\partial \xi} \\
\frac{\partial N_{i}}{\partial \eta}
\end{array}\right] \frac{\partial \psi}{\partial s}\right. \\
& \left.+\frac{\partial}{\partial s}\left[\begin{array}{c}
\frac{\partial \psi}{\partial \xi} \\
\frac{\partial \psi}{\partial \eta}
\end{array}\right] N_{i}+\left[\begin{array}{c}
\frac{\partial \psi}{\partial \xi} \\
\frac{\partial \psi}{\partial \eta}
\end{array}\right]\left(\frac{\partial N_{i}}{\partial \xi} \frac{\partial \xi}{\partial s}+\frac{\partial N_{i}}{\partial \eta} \frac{\partial \eta}{\partial s}\right)\right)
\end{aligned}
$$


Working with XFEM, the derivative of the first Jacobian $\mathbf{J}_{1}$ is equal to zero and the derivative of the XFEM part of the B matrix, as given in Eq.(24), is reduced to the following equation:

$$
\begin{array}{r}
\frac{\partial}{\partial s}\left[\begin{array}{c}
\frac{\partial N_{i}^{\star}}{\partial x} \\
\frac{\partial N_{i}^{\star}}{\partial y}
\end{array}\right]=\mathbf{J}_{1}^{-1}\left(\left[\begin{array}{c}
\frac{\partial^{2} N_{i}}{\partial \xi^{2}} \frac{\partial \xi}{\partial s}+\frac{\partial^{2} N_{i}}{\partial \xi \partial \eta} \frac{\partial \eta}{\partial s} \\
\frac{\partial^{2} N_{i}}{\partial \eta^{2}} \frac{\partial \eta}{\partial s}+\frac{\partial^{2} N_{i}}{\partial \eta \partial \xi} \frac{\partial \xi}{\partial s}
\end{array}\right] \psi+\left[\begin{array}{c}
\frac{\partial N_{i}}{\partial \xi} \\
\frac{\partial N_{i}}{\partial \eta}
\end{array}\right] \frac{\partial \psi}{\partial s}\right. \\
\left.+\frac{\partial}{\partial s}\left[\begin{array}{c}
\frac{\partial \psi}{\partial \xi} \\
\frac{\partial \psi}{\partial \eta}
\end{array}\right] N_{i}+\left[\begin{array}{c}
\frac{\partial \psi}{\partial \xi} \\
\frac{\partial \psi}{\partial \eta}
\end{array}\right]\left(\frac{\partial N_{i}}{\partial \xi} \frac{\partial \xi}{\partial s}+\frac{\partial N_{i}}{\partial \eta} \frac{\partial \eta}{\partial s}\right)\right)
\end{array}
$$

In equations (18), (22) and (25), the following terms appear: $\frac{\partial \xi}{\partial s}$ and $\frac{\partial \eta}{\partial s}$. These terms accounts for the variation of the position of the points in the first parametric space $(\xi, \eta)$ with the design parameter $s$. They can be interpreted as a velocity field $V$. Computing the velocity field is an important issue and the practical methods used to evaluate it are detailed in the next section.

\section{VELOCITY FIELD COMPUTATION}

In classical shape optimization, the knowledge of the velocity field is necessary to perform the sensitivity analysis. The velocity field describes the first order update of the mesh node positions with respect to a small perturbation of the boundary design variables. From a theoretical point of view [13], the velocity field remains partly arbitrary provided that it matches the prescribed displacements at the material interface boundaries. That is the velocity field is assigned to the modification of the material interfaces. Starting from its prescribed value on the boundary, the velocity field can be extended in the interior of the domain. This extension must remains sufficiently smooth. This condition is satisfied with the same smoothness properties as the displacement field. Since the sensitivity analysis is a key issue in shape optimization, computing an accurate velocity field is a critical operation. Therefore, a lot of efforts were focused on velocity field computation and many procedures were developed.

This work focuses on two methods to deal with the velocity field: the finite difference method and an original method inspired from the boundary displacement method.

\subsection{Finite difference approach to determine the velocity field}

To compute the velocity field, a finite difference on the intersection location of the material interface before $(a(s), b(s))$ and after $(a(s+\delta s), b(s+\delta s))$ perturbation can be applied as shown in Figure 7. This method is straightforward and easy to implement.
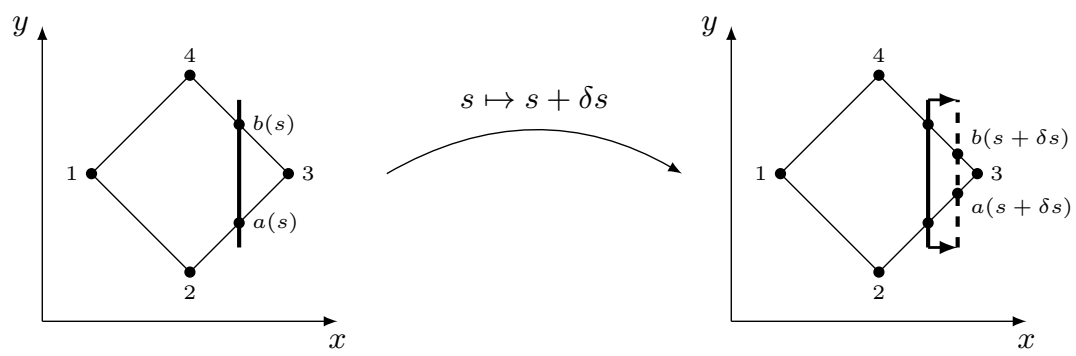

Figure 7. Evaluation of the velocity field by finite differences. 
The velocity field values at the intersections $a$ and $b$ are evaluated by finite differences as expressed in Eq.(26). Since the mesh is fixed, it sounds natural to extend the velocity field over the thin boundary layer made of the XFEM across the material interface. Over this boundary layer, the velocity field is set to zero. It comes that the velocity field values at the nodes belonging to the parent element are set to zero.

$$
\text { On the boundary } \begin{cases}V_{\xi_{i}}=\frac{d \xi_{i}}{d s}=\frac{\xi_{i}(s+\delta s)-\xi_{i}(s)}{\delta s}, & i=a, b \\ V_{\eta_{i}}=\frac{d \eta_{i}}{d s}=\frac{\eta_{i}(s+\delta s)-\eta_{i}(s)}{\delta s}, & i=a, b .\end{cases}
$$

Inside the domain, a virtual displacement field is used. This one results from the finite element interpolation of the background mesh used for Gauss integration on XFEM. Finite element shape function insures a sufficient smoothness of the velocity field while the computation of the local value can be performed easily with a low computational effort.

$$
\text { Inside the domain }\left\{\begin{array}{l}
V_{\xi}=\frac{d \xi}{d s}=\sum_{i} N_{i}(l, m) V_{\xi_{i}}, \\
V_{\eta}=\frac{d \eta}{d s}=\sum_{i} N_{i}(l, m) V_{\eta_{i}} .
\end{array}\right.
$$

This method holds as long as there remains at least one intersection between the element edges and the iso-zero level set, i.e. there is no change of element status. If there is no intersection left, applying the finite difference method is no longer possible for the material interface. Whenever this case occurs, the velocity field on the interface can not be computed. Then the strategies developed for the finite differences, as detailed in section 3.2.1, can be applied.

\subsection{Adapted boundary displacement method}

To avoid finite difference computations, a method, inspired from the boundary displacement method, is developed. The boundary displacement method consists in evaluating the velocity field by solving an auxiliary problem with prescribed interface displacements. The proposed method is adapted to the XFEM framework and an extra problem has to be solved for each element initially cut by the interface.

The subdivision of an element can be performed introducing extra inner nodes in the integration mesh or not, as shown in Figure 8. Choosing either way leads to different auxiliary problems to solve. The formulations obtained in each case are given in Table I, where $\widetilde{\mathbf{K}}$ is a stiffness matrix related to the auxiliary problem, $\overline{\mathbf{V}}$ collects the prescribed displacements on the interface and the zero displacement on the nodes belonging to the parent element, $\mathbf{V}$ are the displacements of the integration mesh inner nodes.

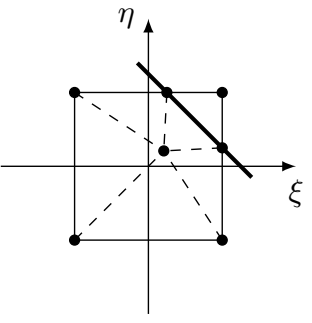

(a) With an inner node.

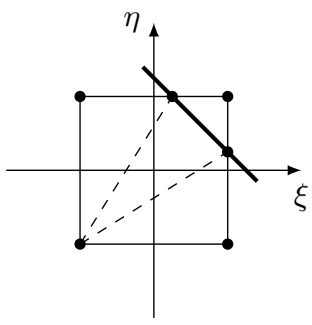

(b) Without inner node.

Figure 8. Subdivision strategy with or without introducing extra inner nodes. 
Table I. Auxiliary problem formulation

\begin{tabular}{lc}
\hline With extra inner nodes & {$\left[\begin{array}{cc}\widetilde{\mathbf{K}}_{\overline{V V}} & \widetilde{\mathbf{K}}_{\bar{V} V} \\
\widetilde{\mathbf{K}}_{V \bar{V}} & \widetilde{\mathbf{K}}_{V V}\end{array}\right]\left[\begin{array}{c}\overline{\mathbf{V}} \\
\mathbf{V}\end{array}\right]=\left[\begin{array}{l}0 \\
0\end{array}\right]$} \\
Without extra inner node & {$\left[\widetilde{\mathbf{K}}_{\overline{V V}}\right][\overline{\mathbf{V}}]=0$} \\
\hline
\end{tabular}

To solve the auxiliary problem, the related stiffness matrix $\widetilde{\mathbf{K}}$ has to be evaluated. Since this matrix is different from the elementary one, this can lead to an extra cost. However, if no inner node is introduced, the displacements at each node are already known and no extra computation is needed. For this reason, in this paper, the subdivision is achieved without introducing extra nodes, even if it could lead to less regular subelements.

To apply this adapted boundary displacement method, appropriate interface displacements have to be prescribed. As the interface is represented by the iso-zero level set, the level set description can be advantageously exploited to compute the velocity field on the interface.

As explained in section 2.5.1, the level set function is approximated by a straight line within each XFEM element to find the interface location. This approximation provides an analytical expression of the coordinates of the intersections. To obtain the velocity field on the interface, the equation of the coordinates Eq. (8) is derived with respect to the design parameter $s$, leading to the following results:

$$
\frac{d}{d s}\left[\begin{array}{c}
\xi^{\star} \\
\eta^{\star}
\end{array}\right]=\frac{d}{d s}\left[\begin{array}{c}
t \xi_{2}+(1-t) \xi_{1} \\
t \eta_{2}+(1-t) \eta_{1}
\end{array}\right]=\left[\begin{array}{c}
\frac{d t}{d s}\left(\xi_{2}-\xi_{1}\right) \\
\frac{d t}{d s}\left(\eta_{2}-\eta_{1}\right)
\end{array}\right]
$$

with

$$
\frac{d t}{d s}=\frac{d}{d s}\left(\frac{\left|\phi_{1}\right|}{\left|\phi_{1}\right|+\left|\phi_{2}\right|}\right) .
$$

The derivative of $t$ requires the computation of the derivatives of the level set nodal values. For the sake of simplicity, these derivatives are here evaluated by finite differences, since it is always possible regardless of the change of the element status.

Since the velocity field nodal values are known, the velocity field values can be recovered anywhere within the subelements using a classical FEM approximation in the second parametric space, as expressed in Eq.(27).

\section{NUMERICAL VALIDATION}

The analytical computation of the stiffness matrix derivative is now validated against numerical examples. The results are compared to those provided by global finite differences and the semianalytical approach.

\subsection{Bar made of quadrangular elements}

The first test case focuses on a structure made of five bilinear Q4 quadrangles assembled to form a bar-like shape, as shown in Figure 9. The left side of the structure is fixed in both $x$ and $y$ directions, while the right side is submitted to an uniform distributed force $f$. The structure is divided in two subdomains $\Omega_{A}$ and $\Omega_{B}$, filled with two materials A and B, exhibiting different properties. All the parameters used in this test case are summarized in Table II.

In this test case, the sensitivity of the compliance of the structure is evaluated for successive locations of the material interface. The external forces are assumed independent of the design parameter $s$ and the derivative of the compliance $C$ is expressed as [8]:

$$
\frac{d C}{d s}=-\mathbf{u}^{T} \frac{\partial \mathbf{K}}{\partial s} \mathbf{u}
$$




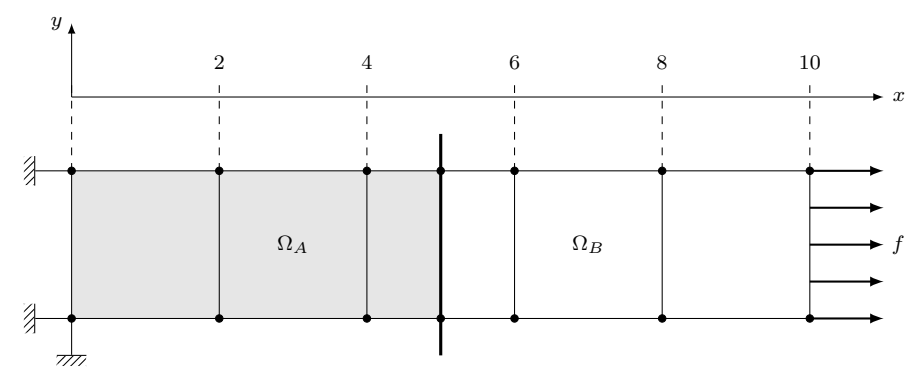

Figure 9. Validation test case: bimaterial bar geometry, boundary conditions and finite element mesh.

Table II. Validation test case: parameters.

\begin{tabular}{lll}
\hline Dimensions & {$[\mathrm{m}]$} & $L \times l \times t=10 \times 2 \times 1$ \\
Elastic moduli & {$\left[N / m^{2}\right]$} & $E_{A}=1, E_{B}=10$ \\
Poisson's ratio & {$[-]$} & $\nu_{A}=\nu_{B}=0$ \\
Distributed load & {$[N / m]$} & $f=1$ \\
Level set function & & $\phi(\mathbf{x}, s)=x-s$ \\
Mesh & $5 \times 1$ bilinear Q4 \\
Gauss points per subelement & & $n_{g p}=7$ \\
\hline
\end{tabular}

Test case results The compliance derivative is evaluated using the three following approaches: a global finite difference computation (FD), a semi-analytical approach (SA) and the analytical approach developed in this paper (A). Moreover, as the Poisson's ratio is equal to zero, the structure behaves as a 1D bar and an exact solution can be computed. All the used equations are reminded in Table III.

Table III. Summary of the sensitivity analysis approaches used.

\begin{tabular}{ll}
\hline Finite difference (FD) & $\frac{d \mathcal{C}}{d s} \simeq \frac{\mathcal{C}(s+\delta s)-\mathcal{C}(s)}{\delta s}, \delta s=10^{-4} s$ \\
Semi-analytical (SA) & $\frac{d \mathbf{K}}{d s} \simeq \frac{\mathbf{K}(s+\delta s)-\mathbf{K}(s)}{\delta s}, \delta s=10^{-4} s$ \\
Analytical (A) & $\frac{\partial \mathbf{K}}{\partial s} \simeq \frac{\partial}{\partial s}\left(\sum_{g p} w \mathbf{B}^{T} \mathbf{H ~ B ~}\left|\mathbf{J}_{1}\right|\left|\mathbf{J}_{2}\right|\right)$ \\
\hline
\end{tabular}

The values of the compliance derivative for various locations $s$ of the interface are given in Table IV as well as the exact analytical solution. One can remark that the results coming from the three different approaches are in excellent agreement. Furthermore, the errors with respect to the exact analytical solution is very small. Focussing on the central element, similar results are found and are represented in Figure 10.

Focusing on the behavior of the three approaches near the boundary between elements provides more interesting conclusions. Table V shows that the analytical approach developed allows to deal with the sensitivity analysis even if the interface is very close to the boundary between elements while other approaches based on finite differences fall into trouble. Using the analytical approach allows to widen the range where the sensitivity analysis can be performed. However, when the interface location coincides with some mesh nodes, the sensitivity analysis can not be performed. This drawback is inherent to the XFEM approach that was chosen to perform the analysis.

Varying the test case parameters The same test case is used but setting the Poisson's ratio value from 0 to 0.3 . As the Poisson's ratio value differs from zero, the structure doesn't behave as a 1D bar anymore and no exact analytical results is available. The compliance derivative values for various 
Table IV. Test case results for $\nu=0$.

\begin{tabular}{lllll}
$s$ & Exact & FD central & SA central & A \\
\hline 2.5 & -1.8 & -1.799999 & -1.800000 & -1.799999 \\
3 & -1.8 & -1.800000 & -1.800000 & -1.800000 \\
3.5 & -1.8 & -1.799999 & -1.800000 & -1.800000 \\
4.5 & -1.8 & -1.800000 & -1.800000 & -1.799999 \\
5 & -1.8 & -1.799999 & -1.800000 & -1.799999 \\
5.5 & -1.8 & -1.799999 & -1.800001 & -1.799999 \\
6.5 & -1.8 & -1.800000 & -1.800001 & -1.800000 \\
7 & -1.8 & -1.800000 & -1.800001 & -1.800000 \\
7.5 & -1.8 & -1.799999 & -1.800002 & -1.800000
\end{tabular}

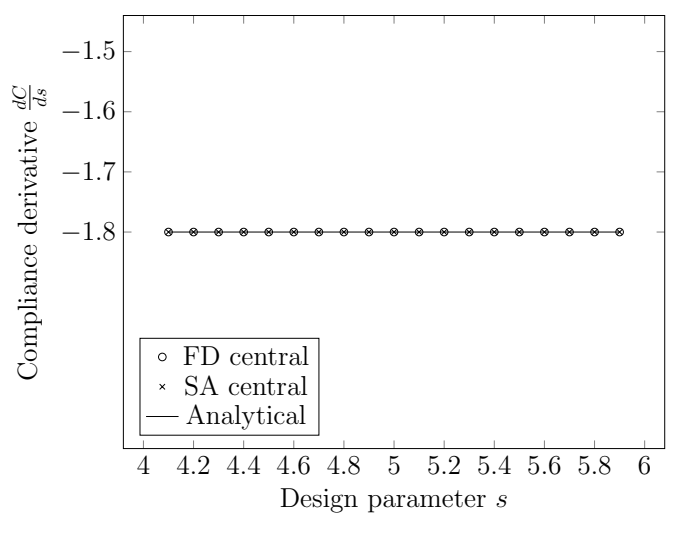

Figure 10. Test case results on the central element for $\nu=0$.

Table V. Test case results for $\nu=0$ near one element boundary.

\begin{tabular}{lllll}
$s$ & Exact & FD forward & FD backward & FD central \\
\hline 3.999 & -1.8 & -1.800000 & -1.800000 & -1.800000 \\
3.9992 & -1.8 & -1.799999 & -1.799999 & -1.799999 \\
3.9994 & -1.8 & -1.800000 & -1.800000 & -1.800000 \\
3.9996 & -1.8 & -1.800000 & -1.799999 & -1.800000 \\
3.9997 & -1.8 & -1.800000 & -1.800000 & -1.800000 \\
3.9998 & -1.8 & -1.800000 & -1.799999 & -1.799999 \\
3.9999 & -1.8 & -1.799999 & -1.800000 & -1.799999 \\
& & & & \\
$s$ & SA forward & SA backward & SA central & $\mathrm{A}$ \\
\hline 3.999 & -1.803238 & -1.796762 & -1.800000 & -1.800000 \\
3.9992 & -1.803238 & -1.796762 & -1.800000 & -1.799999 \\
3.9994 & -1.803239 & -1.796761 & -1.800000 & -1.799999 \\
3.9996 & -1.803239 & -1.796761 & -1.800000 & -1.800000 \\
3.9997 & $/ *$ & -1.796761 & $/ *$ & -1.800000 \\
3.9998 & $/ *$ & -1.796761 & $/ *$ & -1.799999 \\
3.9999 & $/ *$ & -1.796760 & $/ *$ & -1.799999 \\
& \multirow{2}{*}{$*$ Procedure is unable to compute the sensitivity. }
\end{tabular}

locations $s$ of the interface are given in Table VI. The sensitivity results from the three approaches (FD, SA and A) are again in excellent agreement. Focussing on the central element, similar results are found and are represented in Figure 11.

Focusing on the behavior of the three approaches near the boundary between elements, Table VII shows again that the analytical approach allows to expand the domain in which the sensitivity analysis can be performed without any trouble or technical difficulties. One can also remark that the finite difference and the semi-analytical approach give erroneous results very close to the boundary between elements and that some preprocessing strategies have to be applied.

\subsection{Shape optimization of a stiff and a soft inclusion in an infinite plate}

To illustrate the efficiency of the developed analytical approach to perform the sensitivity analysis, a classical shape optimization problem is investigated. The problem aims at finding the optimal elliptical shape of a stiff and a soft inclusions in a plate. 
Table VI. Test case results for $\nu=0.3$.

\begin{tabular}{llll}
$s$ & FD central & SA central & A \\
\hline 2.5 & -1.7952710 & -1.7952712 & -1.7952710 \\
3 & -1.7864811 & -1.7864814 & -1.7864811 \\
3.5 & -1.7990212 & -1.7990217 & -1.7990212 \\
4.5 & -1.7950962 & -1.7950969 & -1.7950962 \\
5 & -1.7864488 & -1.7864496 & -1.7864488 \\
5.5 & -1.7989892 & -1.7989905 & -1.7989892 \\
6.5 & -1.7950783 & -1.7950798 & -1.7950783 \\
7 & -1.7864612 & -1.7864629 & -1.7864612 \\
7.5 & -1.7980988 & -1.7981011 & -1.7980987
\end{tabular}

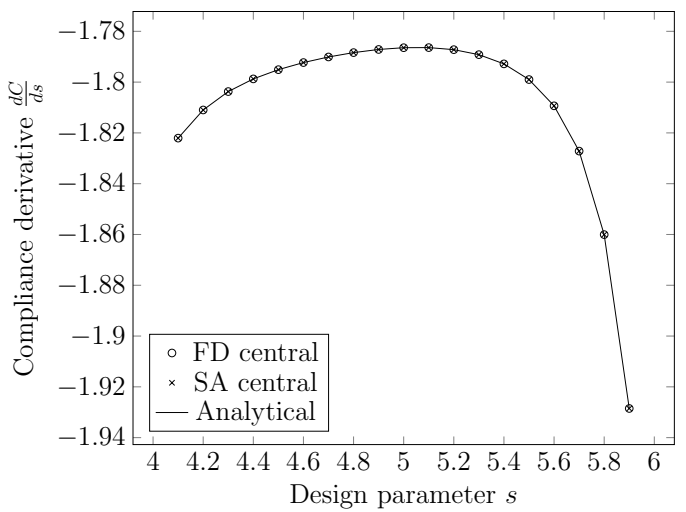

Figure 11. Test case results on the central element for $\nu=$ 0.3 .

Table VII. Test case results for $\nu=0.3$ near one element boundary.

\begin{tabular}{lllll}
$s$ & FD forward & FD backward & FD central & \\
\hline 3.999 & -2.1046103 & -2.1034229 & -2.1040166 & \\
3.9992 & -2.1052065 & -2.1040159 & -2.1046112 & \\
3.9994 & -2.1058042 & -2.1046105 & -2.1052074 & \\
3.9996 & -2.1064036 & -2.1052066 & -2.1058051 & \\
3.9997 & 73.6810921 & -2.1055053 & 35.7877934 & \\
3.9998 & 73.7457260 & -2.1058043 & 35.8199608 & \\
3.9999 & 73.8104376 & -2.1061037 & 35.8521669 & \\
& & & & A \\
$s$ & SA forward & SA backward & SA central & -2.10401559 \\
3.999 & -6.8337315 & 2.6245374 & -2.1045970 & -2.10461021 \\
3.9992 & -9.4257274 & 5.2146782 & -2.1055245 & -2.10520638 \\
3.9994 & -14.9996938 & 10.7860069 & -2.1068434 & -2.10580409 \\
3.9996 & -30.8509364 & 26.6319040 & -2.1095162 & -2.10610352 \\
3.9997 & $/ *$ & 48.7483057 & $/ *$ & -2.10640334 \\
3.9998 & $/ *$ & 111.7920428 & $/ *$ & -2.10670356 \\
3.9999 & $/ *$ & 451.40897744 & $/ *$ &
\end{tabular}

Description of the problem The problem is symmetric and only a quarter of the plate is studied. The geometry as well as the boundary conditions applied are summarized on Figure 12. A plane stress state is assumed.

The optimization problem consists in finding the optimal shape of a stiff inclusion. An elliptical shape is assumed. The design parameters are the semi-axes of the ellipse $\mathbf{s}^{T}=[a b]$. The compliance of the structure is minimized, while the volume of the inclusion remains smaller than a given bound $V_{\max }$. No other constraint is prescribed, except some side constraints on the design parameters. The convergence criterion chosen is the variation of the objective function. This relative variation must be smaller than $10^{-3}$. Two different meshes are used: a $50 \times 50 \mathrm{Q} 4$ quadrangular mesh (element size $=0.2$ ) and an equivalent $\mathrm{T} 3$ triangular mesh (element size $\simeq 0.2$ ) presented in Figure 13.The optimization problem is solved by the Method of Moving Asymptotes (MMA) of Svanberg [25]. All the parameters used through the optimization process are listed in Table VIII. Finally, the 


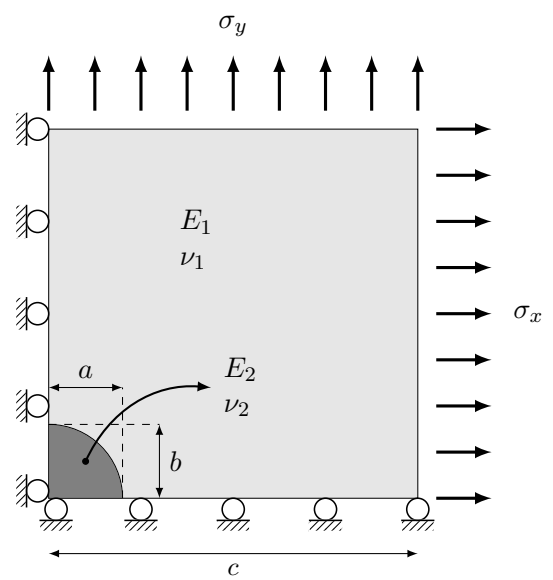

Figure 12. Finding the optimal shape of a stiff inclusion in an infinite plate.

optimization problem is stated as follow:

$$
\begin{array}{ll}
\min _{\mathbf{s}} & \mathcal{C}=\mathbf{u}^{T} \mathbf{K} \mathbf{u}, \\
\text { s.t. } & V_{\text {incl }} \leq V_{\max } \\
& \underline{\mathbf{s}} \leq \mathbf{s} \leq \overline{\mathbf{s}}
\end{array}
$$

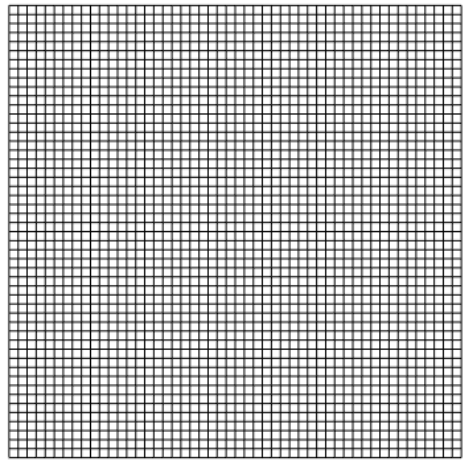

(a) Structured mesh with Q4 (element size $=0.2$ ).

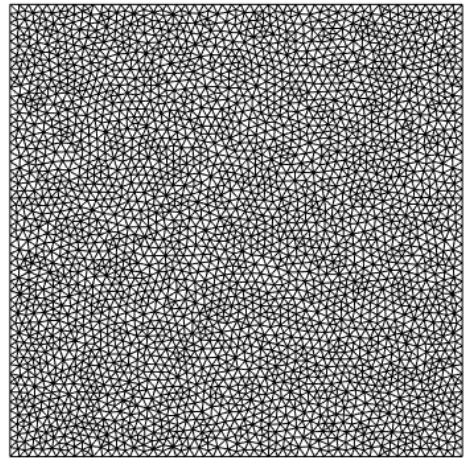

(b) Unstructured mesh with T3 (element size $\simeq 0.2$ ).

Figure 13. Stiff inclusion in an infinite plate: meshes.

XFEM integration validation: Number of Gauss points per subelement To emphasize the importance of using a sufficient number of Gauss points to perform the integration with XFEM, the optimization problem of a stiff inclusion in an infinite plate, described in Figure 12 and Table VIII, is used. The analysis is performed on the structured mesh presented in Figure 13. The analysis and the sensitivity analysis for the initial configuration of the problem are carried out. The latter is performed using the global finite differences (FD), the semi-analytical approach (SA) and the developed analytical approach (A). One, three, seven and twelve Gauss points per subelement are successively used. The results, i.e. the compliance and its derivatives, are shown in Table IX. One Gauss point is definitely not sufficient to capture discontinuous behaviors around the interface. With twelve Gauss points, the results are really expensive for no further improvement observed in the results. Seven Gauss points seems to be a good compromise between computational efficiency and precision. 
Table VIII. Stiff inclusion in an infinite plate: parameters.

\begin{tabular}{lll}
\hline Dimensions & {$[\mathrm{m}]$} & $c=10, t=1$ \\
Elastic moduli & {$\left[N / m^{2}\right]$} & $E_{1}=1, E_{2}=10$ \\
Poisson's ratio & {$[-]$} & $\nu_{1}=\nu_{2}=0.3$ \\
Distributed loads & {$[N / m]$} & $\sigma_{x}=1, \sigma_{y}=0.5$ \\
Bound on $V_{\text {incl }}$ & {$\left[m^{2}\right]$} & $V_{\max }=2$ \\
Bounds on design variables & {$[m]$} & $0 \leq a \leq 4,0 \leq b \leq 3$ \\
Level set function & & $\phi(\mathbf{x}, \mathbf{s})=\left(\frac{x}{a}\right)^{2}+\left(\frac{y}{b}\right)^{2}-1$ \\
Gauss points per subelement & & $n_{g p}=7$ \\
Convergence criterion & & $\Delta \mathcal{C} \leq 10^{-3}$ \\
\hline
\end{tabular}

Table IX. Sensitivity of the developed analytical approach to the number of Gauss points used per subelement.

\begin{tabular}{llllll} 
GP & Compliance & s & FD central & SA central & A \\
\hline 1GP & 94.78122 & $a$ & -0.5160554 & -0.5160557 & -0.5159780 \\
& & $b$ & -0.3859520 & -0.3859522 & -0.3858941 \\
\hline 3GP & 94.76729 & $a$ & -0.5774089 & -0.5774091 & -0.5773223 \\
& & $b$ & -0.3709876 & -0.3709877 & -0.3709319 \\
\hline 7GP & 94.76728 & $a$ & -0.5771527 & -0.5771529 & -0.5770661 \\
& & $b$ & -0.3710224 & -0.3710225 & -0.3709667 \\
\hline 12GP & 94.76728 & $a$ & -0.5771497 & -0.5771499 & -0.5770632 \\
& & $b$ & -0.3710101 & -0.3710102 & -0.3709545
\end{tabular}

Validation of the developed analytical approach The developed analytical approach is validated by evaluating the sensitivity of the initial configuration of the problem with respect to the design parameters s. The derivative of the objective function is evaluated with the three following approaches on the structured and unstructured meshes: the global finite differences (FD), the semianalytical approach (SA) and the developed analytical approach (A). The results are listed in Table $\mathrm{X}$. The three approaches are clearly in great agreement.

Table X. Validation of the developed analytical approach by evaluation of the sensitivity of the initial configuration of the problem with respect to the design parameters $\mathbf{s}$.

\begin{tabular}{llllll} 
Mesh & s & FD central & SA central & A & Error A-FD (\%) \\
\hline Structured Q4 & $a$ & -0.5771527 & -0.5771529 & -0.5770661 & $1.500510^{-2}$ \\
& $b$ & -0.3710224 & -0.3710225 & -0.3709667 & $1.501310^{-2}$ \\
Unstructured T3 & $a$ & -0.5922506 & -0.5922506 & -0.5921618 & $1.499810^{-2}$ \\
& $b$ & -0.3593348 & -0.3593348 & -0.3592809 & $1.501210^{-2}$
\end{tabular}

Problem results The results obtained with the structured and the unstructured meshes are very close. Therefore, the solutions are only presented for the structured mesh. The initial and the optimized configurations of the elliptical inclusion are given in Figure 14. The optimized shape is obtained after 13 iterations. One can see that the stiff inclusion aligns with the applied loads. The obtained configuration becomes similar to a reinforcement fiber in a soft matrix.

The evolution of the objective and the constraint functions are provided in Figure 15. As shown on Figure 15(b), the constraint becomes active after few iterations. On Figure 15(a), one can see that the compliance diminishes from one iteration to another and the optimization process stops as its relative variation between two iterations becomes smaller than $10^{-3}$. If a smaller tolerance on the 


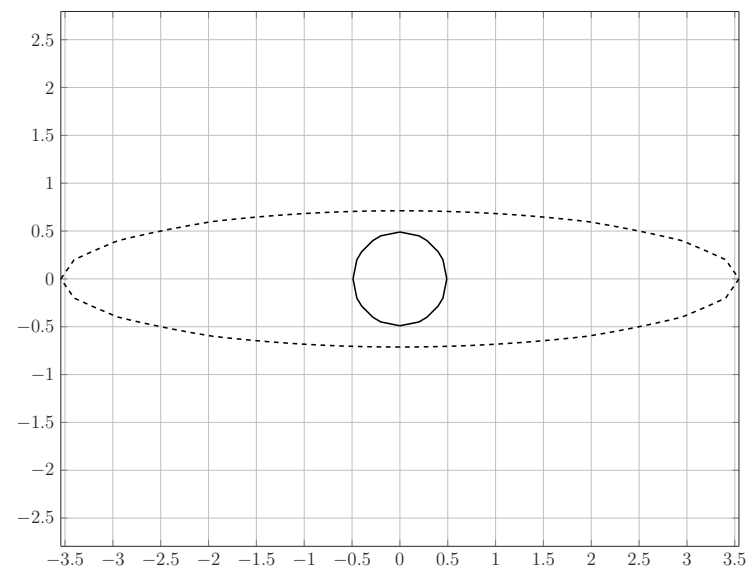

Figure 14. Initial $(a=b=0.5)$ and optimized $(a=3.60, b=0.71)$ elliptical shape of the stiff inclusion in an infinite plate.

compliance variations is chosen, the ellipse tends to form a layer and flattens to align with the loads as $a$ becomes bigger and $b$ smaller.

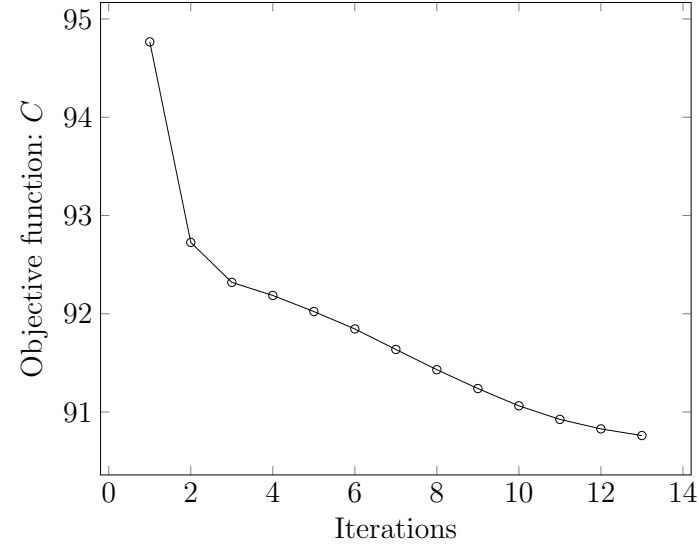

(a) Objective function.

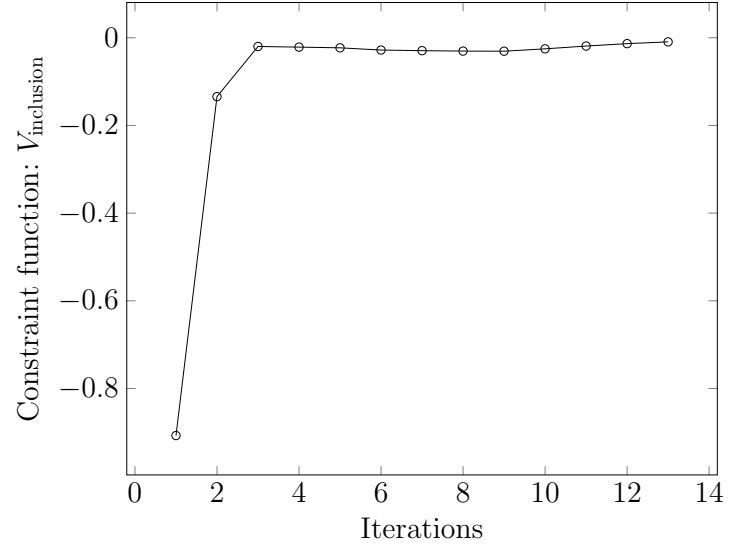

(b) Constraint function.

Figure 15. Evolution of the objective and the constraint functions along the optimization process for the stiff inclusion in an infinite plate.

To observe the convergence process in details, the values of the compliance and the volume are computed over the design domain. Figure 16 shows the path followed iteration after iteration through the optimization process.

Varying the parameters: Soft inclusion The same optimization problem is solved but modifying the elastic moduli of the materials so that the inclusion is now soft $\left(E_{2}=1\right)$ with comparison to the plate $\left(E_{1}=10\right)$. The optimization problem still consists in finding the optimal shape but of a soft inclusion. An elliptical shape is assumed. The optimization problem is now formulated as:

$$
\begin{array}{ll}
\min _{\mathbf{s}} & \mathcal{C}=\mathbf{u}^{T} \mathbf{K} \mathbf{u}, \\
\text { s.t. } & V_{\text {incl }} \geq V_{\min }, \\
& \underline{\mathbf{s}} \leq \mathbf{s} \leq \overline{\mathbf{s}}
\end{array}
$$




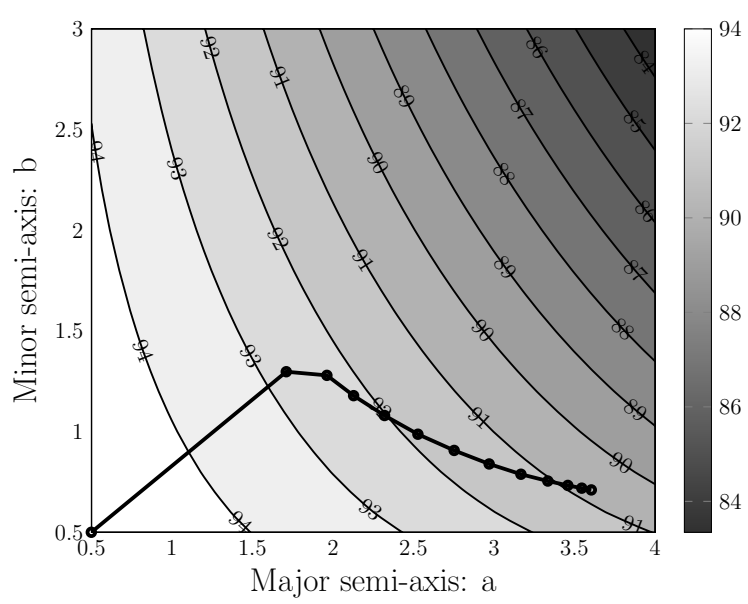

(a) Objective function.

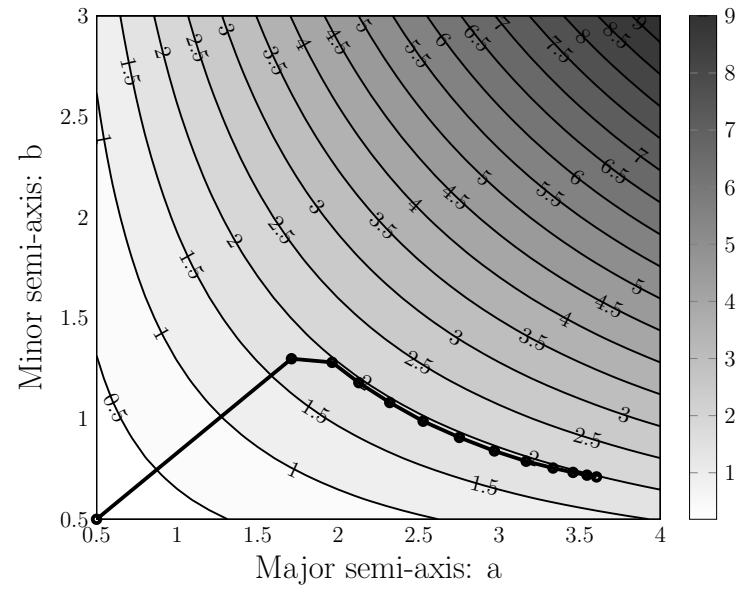

(b) Constraint function.

Figure 16. Iteration path along the optimization process for the stiff inclusion in an infinite plate.

The same results as the ones obtained for the stiff inclusion are provided. Here, only the structured mesh shown in Figure 13 is used. Figure 17 provides the initial and optimized configurations of the elliptical soft inclusion. Figure 18 shows the evolution of the objective and constraint functions. Figure 19 illustrates the convergence path followed through the optimization process. The optimal shape is now an ellipse with main axes aligned with the far field principal stresses. The ratio $\frac{a}{b}$ between the two axes tends to 2 which would be the analytical solution when $E_{\text {soft }}$ tends to zero.

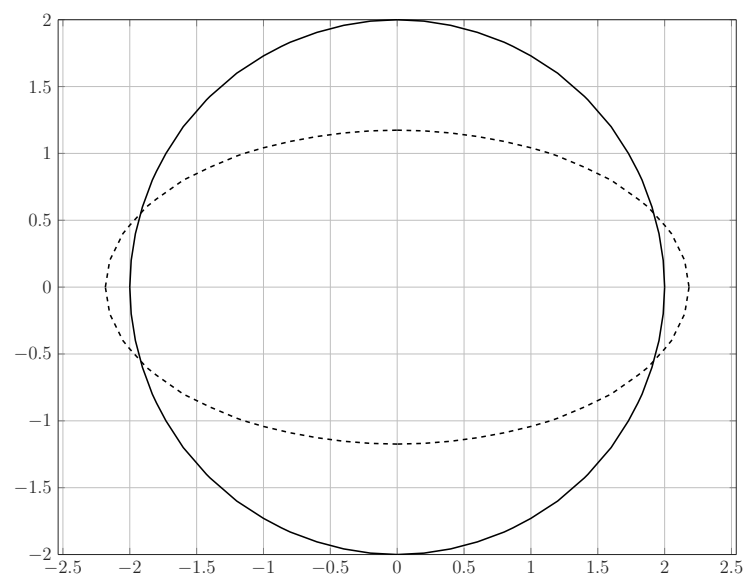

Figure 17. Initial $(a=b=2)$ and optimized $(a=2.183, b=1.176)$ elliptical shape of the soft inclusion in an infinite plate.

\subsection{Dealing with complex geometries using overlapping level set functions}

In general, several level set functions can be used to represent complex geometries in optimization problems. Each of the level set functions are characterized by its own geometric features used as design parameters. Applying boolean operations, as the union, the difference or the complement, a global level set function can be obtained starting from several independent level set functions.

This section demonstrates that multiple or overlapping level set functions can be handled by the developed analytical sensitivity analysis. In fact, during the optimization process, the level set 


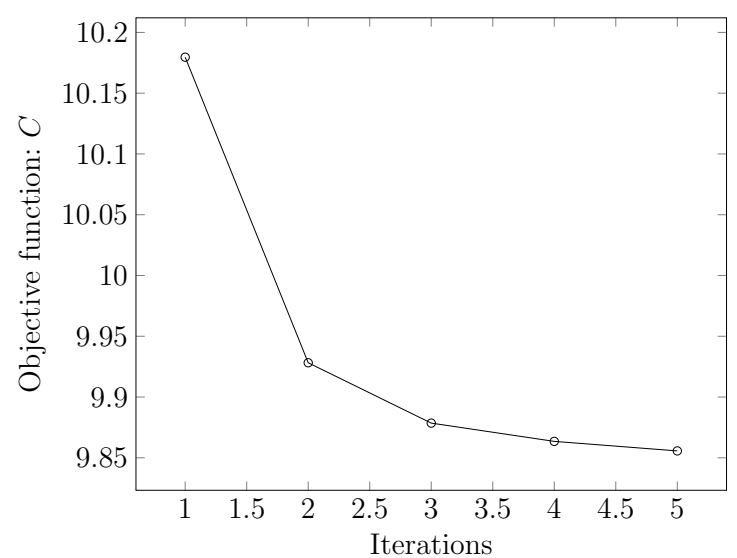

(a) Objective function.

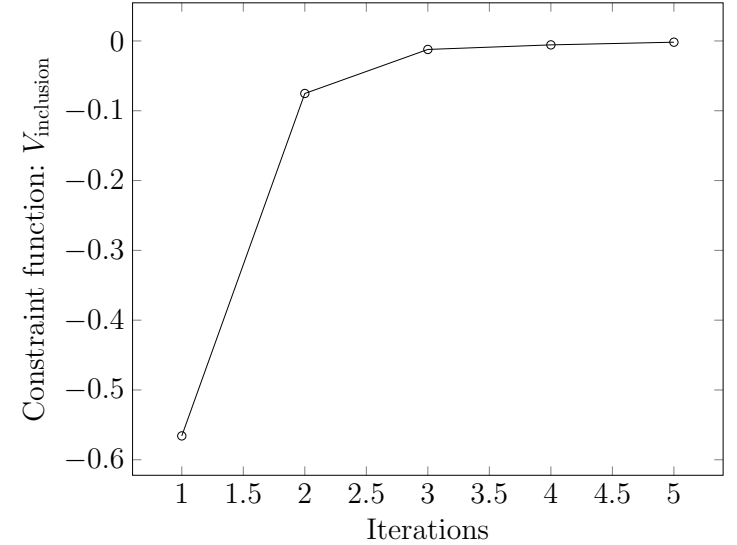

(b) Constraint function.

Figure 18. Evolution of the objective and the constraint functions along the optimization process for the soft inclusion in an infinite plate.

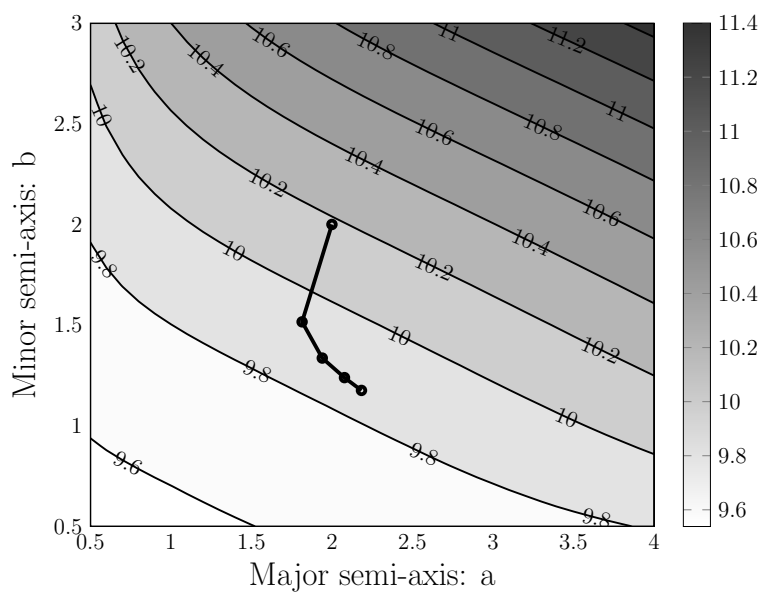

(a) Objective function.

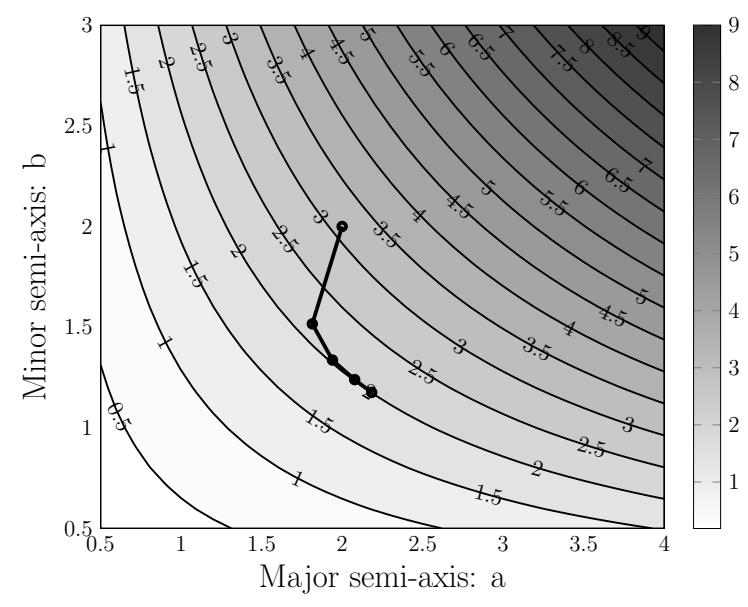

(b) Constraint function.

Figure 19. Iteration path along the optimization process for the soft inclusion in an infinite plate.

functions can overlap or merge. However, performing the sensitivity analysis, the design parameters, i.e. the geometric features of the initial level set functions, are still treated independently. Processing this way, no additional treatment is necessary to carry out the analytical approach.

To illustrate the efficiency of the developed approach when overlapping occurs, the case of two stiff circular inclusions in a plate submitted to tension is studied. The inclusions are described using two distinct level set functions $\phi_{1}$ and $\phi_{2}$ that are combined by the boolean union operation to create a global level set function $\phi_{\text {global }}$.

Description of the problem The geometry of the plate as well as the boundary conditions are provided in Figure 20. A plane stress state is assumed. The problem of finding the inclusions locations along the $y$ axis as well as their radii is addressed. The design parameters are the locations of the inclusions along the $y$ axis as well as their radii: $\mathbf{s}=\left[\begin{array}{llll}s_{1} & R_{1} & s_{2} & R_{2}\end{array}\right]$. The compliance of the structure is minimized, while the total volume of the inclusions must remain smaller than a given bound $V_{\max }$. No other constraint is prescribed, except some side constraints on the design parameters. 
The chosen convergence criterion is the variation of the objective function. The relative variation of the compliance must be smaller than $10^{-3}$. The problem is solved on a $50 \times 50 \mathrm{Q} 4$ quadrangular mesh (element size $=0.2 \mathrm{~m}$ ), identical to the one presented in Figure 13, and using MMA. All parameters used in the optimization process are listed in Table XI. Finally, the optimization problem is formulated as follow:

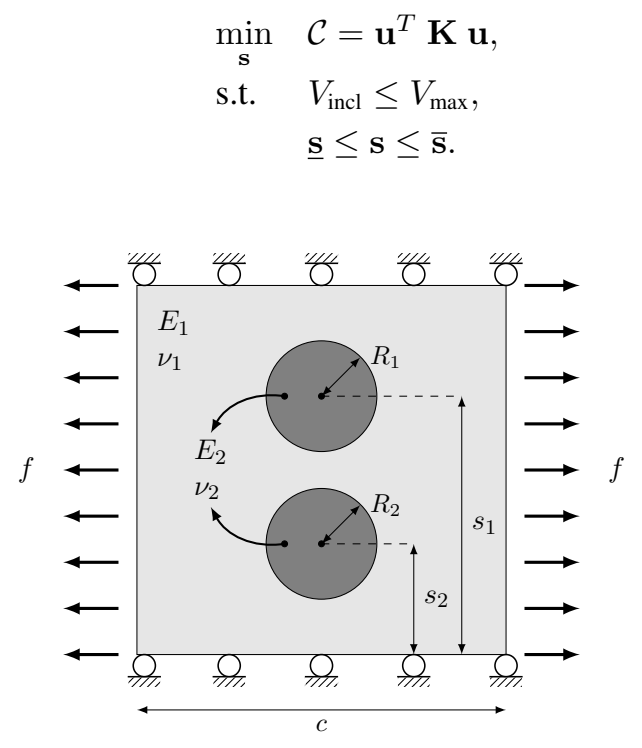

Figure 20. Finding the optimal location and size of two stiff circular inclusions in a plate.

Table XI. Two stiff circular inclusions in a plate: parameters.

\begin{tabular}{lll}
\hline Dimensions & {$[\mathrm{m}]$} & $c=10, t=1$ \\
Elastic moduli & {$\left[N / m^{2}\right]$} & $E_{A}=1, E_{B}=100$ \\
Poisson's ratio & {$[-]$} & $\nu_{A}=\nu_{B}=0.3$ \\
Distributed load & {$[N / m]$} & $f=10^{-1}$ \\
Bound on $V_{\text {incl }}$ & {$\left[\mathrm{m}^{2}\right]$} & $V_{\max }=10$ \\
Bounds on design variables & {$[\mathrm{m}]$} & $4 \leq s_{1}, s_{2} \leq 6 ; 0 \leq R_{1}, R_{2} \leq 2.5$ \\
Level set function & & $\phi_{1}\left(\mathbf{x}, s_{1}\right)=\sqrt{(x-5)^{2}+\left(y-s_{1}\right)^{2}}-R_{1}$ \\
& & $\phi_{2}\left(\mathbf{x}, s_{2}\right)=\sqrt{(x-5)^{2}+\left(y-s_{2}\right)^{2}}-R_{2}$ \\
& $\phi_{\text {global }}(\mathbf{x}, \mathbf{s})=\phi_{1} \cup \phi_{2}=\max \left(\phi_{1}, \phi_{2}\right)$ \\
Gauss points per subelement & & $n_{g p}=7$ \\
Convergence criterion & $\Delta \mathcal{C} \leq 10^{-3}$ \\
\hline
\end{tabular}

Problem results The initial and the optimized configurations for the two stiff circular inclusions are given in Figure 21. The optimized locations along the $y$ axis and the radii are obtained after five iterations. One can observe that the two inclusions overlap through the optimization process.

The evolution of the objective and the constraint functions are provided in Figure 22. In Figure 22(a), the compliance diminishes through the process, that ends as the relative variation of the compliance between two iterations becomes smaller than $10^{-3}$. If a smaller tolerance is chosen, the two inclusions tend to merge to form a unique and bigger stiff inclusion in the middle of the plate. However, when the inclusions are nearly perfectly superposed, the convergence process becomes delicate. In fact, any small modification of the radii of the inclusions leads to a less total superposition of the geometries. This makes the process unstable, until one of the inclusions completely covers the other. Then, the covered inclusion has no influence on the structural design. 


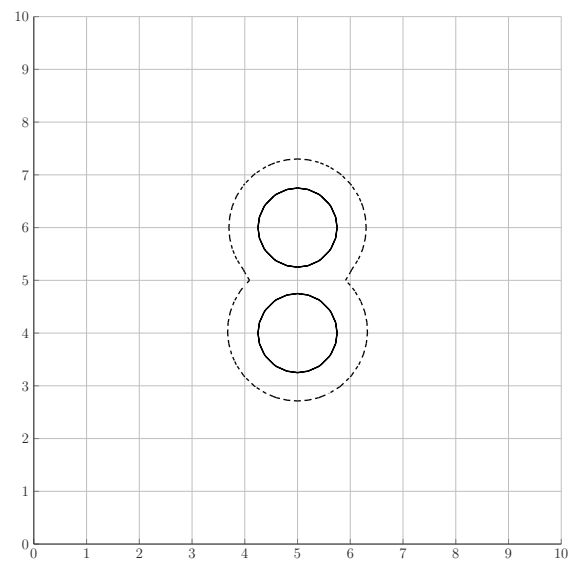

Figure 21. Initial $\left(s_{1}=6, R_{1}=0.75, s_{2}=4, R_{2}=0.75\right)$ and optimized $\left(s_{1}=5.96, R_{1}=1.31, s_{2}=4.04\right.$,

$\left.R_{2}=1.32\right)$ configuration of the two stiff circular inclusions in a plate.

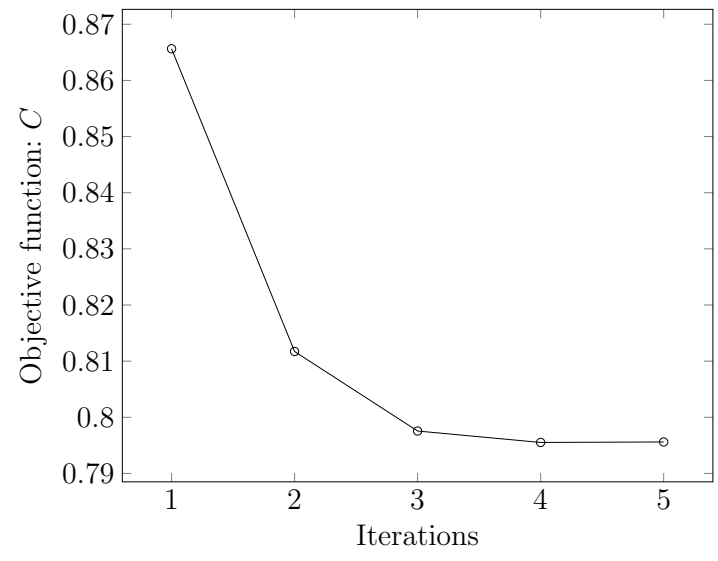

(a) Objective function.

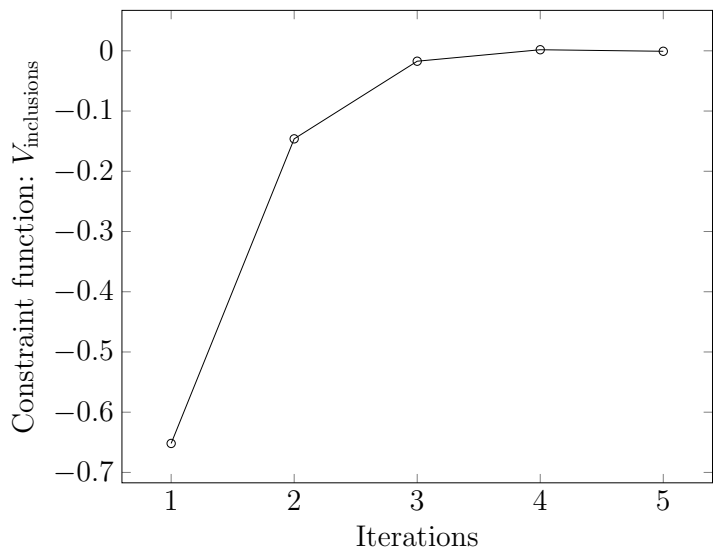

(b) Constraint function.

Figure 22. Evolution of the objective and the constraint functions along the optimization process for the two stiff circular inclusions in a plate.

Validation of the developed analytical approach To show that the developed analytical approach is able to deal with multiple level set functions, the sensitivity analysis with respect to the four design parameters $\mathbf{s}=\left[\begin{array}{lllll}s_{1} & R_{1} & s_{2} & R_{2}\end{array}\right]$ is performed on the initial and the fourth configurations, i.e. the last configuration before convergence of the optimization process, carrying out the three following approaches: the global finite differences (FD), the semi-analytical approach (SA) and the developed analytical approach (A). The obtained results are presented in Figure XII and show that the developed approach is consistent and robust.

\section{CONCLUSION}

This paper has presented an original shape optimization approach of bimaterial structures based on a level set description of geometries and an XFEM discretization. The level set description enables to consider complex boundaries in future work. The XFEM discretization is selected to circumvent the technical difficulty of meshing operations in presence of mobile boundaries as in classical parametric shape optimization. To pave the way for future complex investigations, the 
Table XII. Two stiff circular inclusions in a plate: validation on the initial and fourth configurations.

\begin{tabular}{llllll} 
Configuration & $\mathrm{s}$ & FD central & SA central & A & Error A-FD (\%) \\
\hline 1 & $s_{1}$ & -0.0037668 & -0.0037668 & -0.0037519 & $3.95110^{-1}$ \\
& $R_{1}$ & -0.0556912 & -0.0556912 & -0.0556912 & $1.00110^{-6}$ \\
& $s_{2}$ & 0.0037718 & 0.0037718 & 0.0037817 & $2.63110^{-1}$ \\
& $R_{2}$ & -0.0556936 & -0.0556936 & -0.0556936 & $3.87110^{-7}$ \\
4 & & & & & \\
& $s_{1}$ & -0.0173260 & -0.0173260 & -0.0173143 & $6.74210^{-2}$ \\
& $R_{1}$ & -0.0695147 & -0.0695147 & -0.0695147 & $2.85710^{-7}$ \\
& $s_{2}$ & 0.0179530 & 0.0179530 & 0.0179610 & $4.45910^{-2}$ \\
& $R_{2}$ & -0.0723136 & -0.0723136 & -0.0723136 & $5.35610^{-7}$
\end{tabular}

present work has carefully investigated and validated the sensitivity analysis of the shape internal boundaries between materials.

Without loss of generality, the work assumes that the level sets representing the material interfaces are described by geometrical features whose parameters are considered as the design variables in the optimization process. The developed analytical approach starts from the discretized equations of the linear elasticity problem, $\mathbf{K u}=\mathbf{f}$, and derives it with respect to the design parameters. The sensitivity of the discretized finite element structural responses requires the derivative of the stiffness matrix $\mathbf{K}$.

For classical shape optimization with FEM, the derivative of the stiffness matrix introduces a sensitivity of the physical node positions. In the XFEM framework, the sensitivity analysis is performed on a fixed physical mesh. However, the derivatives introduce a sensitivity of the material subdomains within the XFEM elements and so, a sensitivity of the background mesh used to carry out the integration procedure. Therefore, the mesh flow at the level of physical mesh is avoided, but a velocity field at the level of the XFEM elements has to be considered.

The work explains and details the different terms involved in the stiffness matrix derivative. Then, an original approach to determine the velocity field of material points within the XFEM is proposed. The selected approach takes advantage of the level set representation to determine the prescribed motion of the boundaries. Then, it defines an inner velocity field within the XFEM elements using the background integration mesh and some finite element shape functions to yield a sufficiently smooth velocity field. A simplified procedure is proposed so that no additional problem has to be solved. The latter method proved to be extremely cost efficient while sufficiently smooth to provide accurate results.

The proposed analytical approach was studied and compared with other commonly used ones such as finite differencing or the semi-analytical approach. It was shown that all obtained results are in excellent agreement with each other, but the analytical approach is very efficient and particularly well suited to the chosen XFEM-level set framework. Finally, the analytical approach also proved to be able to cope with difficult situations where the level set is very close to a mesh node. In fact, it still holds while other approaches based on finite perturbation of the level set and finite differences fall into troubles. Even if it looks more complex than finite differencing and the semi-analytical methods, the analytical approach allows performing the sensitivity analysis of structures presenting material interfaces in a systematic way.

Finally the developed analytical sensitivity was illustrated on academic shape optimization problems, where the optimal shape of a material inclusion in an infinite plate is sought. Future work will consider more advanced applications of shape optimization of multiphase structures or microstructures.

\section{ACKNOWLEDGEMENT}

The first author, Lise Noël, is supported by a grant from the Belgian National Fund for Scientific Research (F.R.S.-FNRS) which is gratefully acknowledged. 


\section{REFERENCES}

1. H. M. Adelman and R. T. Haftka. Sensitivity analysis of discrete structural systems. AIAA Journal, 24(5):823-832, 1986.

2. G. Allaire, F. Jouve, and A.-M. Toader. A level-set method for shape optimization. Comptes Rendus Mathmatique, 334(12):1125-1130, 2002.

3. F.-J. Barthold and D. Materna. A modified extended finite element method approach for design sensitivity analysis. International Journal for Numerical Methods in Engineering, 104:209-234, 2015.

4. P. Beckers. Recent developments in shape sensitivity analysis: the physical approach. Engineering Optimization, 18(1-3):67-78, 1991.

5. A.D. Belegundu and S.D. Rajan. Shape optimization approach based on natural design variables and shape functions. Computer Methods in Applied Mechanics and Engineering, 66(1):87-106, 1988.

6. T. Belytschko, C. Parimi, N. Moes, N. Sukumar, and S. Usui. Structured extended finite element methods for solids defined by implicit surfaces. International Journal for Numerical Methods in Engineering, 56(4):609-635, 2003.

7. M. P. Bendsøe and N. Kikuchi. Generating optimal topologies in structural design using a homogenization method. Comput. Methods Appl. Mech. Eng., 71(2):197-224, 1988.

8. M. P. Bendsøe and O. Sigmund. Topology Optimization - Theory, Methods and Applications. Springer, Berlin, 2003.

9. V. Braibant and C. Fleury. Shape optimal design - a performing c.a.d. oriented formulation. In 25th AIAA SDM Conference, 1984.

10. V. Braibant and C. Fleury. Shape optimal design using B-splines. Computer Methods in Applied Mechanics and Engineering, 44(3):247-267, 1984.

11. S. Chen, S. Gonella, W. Chen, and W. K. Liu. A level set approach for optimal design of smart energy harvesters. Computer Methods in Applied Mechanics and Engineering, 199(37-40):2532-2543, 2010.

12. K. K. Choi and K.-H. Chang. A study of design velocity field computation for shape optimal design. Finite Elements in Analysis and Design, 15(4):317 - 341, 1994

13. K. K. Choi and N. H. Kim. Structural Sensitivity Analysis and Optimization: Linear systems. Springer, New York, 2005.

14. R. D. Cook. Finite Element Modeling for Stress Analysis. John Wiley \& Sons, New York, 1995.

15. X. Guo, W. Zhang, and W. Zhong. Stress-related topology optimization of continuum structures involving multiphase materials. Computer Methods in Applied Mechanics and Engineering, 268(0):632 - 655, 2014.

16. L. Li, M. Y. Wang, and P. Wei. Xfem schemes for level set based structural optimization. Frontiers of Mechanical Engineering, 7(4):335-356, 2012.

17. D. Makhija and K. Maute. Numerical instabilities in level set topology optimization with the extended finite element method. Structural and Multidisciplinary Optimization, 49(2):185-197, 2014.

18. N. Moes, M. Cloirec, P. Cartraud, and J.-F. Remacle. A computational approach to handle complex microstructure geometries. Computer Methods in Applied Mechanics and Engineering, 192:3163-3177, 2003.

19. N. Moes, J. Dolbow, and T. Belytschko. A finite element method for crack growth without remeshing. International Journal for Numerical Methods in Engineering, 46(1):131-150, 1999.

20. S. Osher and J. A. Sethian. Fronts propagating with curvature-dependent speed: Algorithms based on hamiltonjacobi formulations. Journal of Computational Physics, 79(1):12-49, 1988.

21. A. Ramani. A pseudo-sensitivity based discrete-variable approach to structural topology optimization with multiple materials. Structural and Multidisciplinary Optimization, 41(4-5):913-934, 2010.

22. M. Rotthaus and F.-J. Barthold. Aspects of sensitivity analysis using the level-set method in structural optimization. PAMM, 7(1):4040041-4040042, 2007.

23. J. Stegmann and E. Lund. Discrete material optimization of general composite shell structures. International Journal for Numerical Methods in Engineering, 62(14):2009-2027, 2005.

24. N. Sukumar, D.L. Chopp, N. Moes, and T. Belytschko. Modeling holes and inclusions by level sets in the extended finite-element method. Computer Methods in Applied Mechanics and Engineering, 190(46-47):6183 - 6200, 2001.

25. K. Svanberg. The method of moving asymptotes-a new method for structural optimization. International Journal for Numerical Methods in Engineering, 24(2):359-373, 1987.

26. S. Torquato and O. Sigmund. Design of materials with extreme thermal expansion using three-phase topology optimization method. Journal of the Mechanics and Physics of Solids, 45(6):1037-1067, 1997.

27. N. P. van Dijk, K. Maute, M. Langelaar, and F. van Keulen. Level-set methods for structural topology optimization: a review. Structural and Multidisciplinary Optimization, 48(3):437-472, 2013.

28. F. van Keulen, R.T. Haftka, and N.H. Kim. Review of options for structural design sensitivity analysis. Part 1: Linear systems. Computer Methods in Applied Mechanics and Engineering, 194(3033):3213-3243, 2005.

29. L. Van Miegroet. Generalized shape optimization using XFEM and level set description. PhD thesis, University of Liège, Liège, 2012.

30. L. Van Miegroet and P. Duysinx. Stress concentration minimization of $2 \mathrm{~d}$ filets using $\mathrm{x}$-fem and level set description. Structural and Multidisciplinary Optimization, 33(4-5):425-438, 2007.

31. N. Vermaak, G. Michailidis, G. Parry, R. Estevez, G. Allaire, and Y. Brechet. Material interface effects on the topology optimization of multi-phase structures using a level set method. Structural and Multidisciplinary Optimization, 50(4):623-644, 2014.

32. H. Waisman. An analytical stiffness derivative extended finite element technique for extraction of crack tip strain energy release rates. Engineering Fracture Mechanics, 77(16):3204 - 3215, 2010.

33. M. Y. Wang and X. Wang. Color level sets: a multi-phase method for structural topology optimization with multiple materials. Computer Methods in Applied Mechanics and Engineering, 193(6-8):469 - 496, 2004.

34. M. Y. Wang, X. Wang, and D. Guo. A level set method for structural topology optimization. Computer Methods in Applied Mechanics and Engineering, 192(1-2):227-246, 2003. 
35. X. Wang, Y. Mei, and M.Y. Wang. Level-set method for design of multi-phase elastic and thermoelastic materials. International Journal for Numerical Methods in Engineering, 1:213-239, 2004.

36. P. Wei, M. Y. Wang, and X. Xing. A study on $\mathrm{x}$-fem in continuum structural optimization using a level set model. Computer-Aided Design, 42(8):708-719, 2010.

37. L. Yin and G.K. Ananthasuresh. Topology optimization of compliant mechanisms with multiple materials using a peak function material interpolation scheme. Structural and Multidisciplinary Optimization, 23(4-5):49-62, 2001. 